\title{
The chemical evolution of a Milky Way-like galaxy: the importance of a cosmologically motivated infall law
}

\author{
E. Colavitti ${ }^{1}$, F. Matteucci ${ }^{1,2}$, and G. Murante ${ }^{3}$ \\ 1 Dipartimento di Astronomia, Università di Trieste, via G. B. Tiepolo 11, 34143 Trieste (TS), Italy \\ e-mail: colavitti@oats.inaf.it \\ 2 INAF - Osservatorio Astronomico di Trieste, via G. B. Tiepolo 11, 34143 Trieste (TS), Italy \\ ${ }^{3}$ INAF - Osservatorio Astronomico di Torino, Strada Osservatorio 20, 10025 Pino Torinese (TO), Italy
}

Received 17 January 2008 / Accepted 13 February 2008

\begin{abstract}
Aims. We aim to find a cosmologically motivated infall law to understand if the $\Lambda \mathrm{CDM}$ cosmology can reproduce the main chemical characteristics of a Milky Way-like spiral galaxy.

Methods. We test several different gas infall laws, starting from that suggested in the two-infall model for the chemical evolution of the Milky Way, but focusing on laws derived from cosmological simulations which follows a concordance $\Lambda$ CDM cosmology. By means of a detailed chemical evolution model for the solar vicinity, we study the effects of the different gas infall laws on the abundance patterns and the G-dwarf metallicity distribution.

Results. The cosmological gas infall law, derived from dark matter halos having properties compatible with the formation of a disk galaxy like the Milky Way, and assuming that the baryons assemble like dark matter, resembles the infall law suggested by the twoinfall model. In particular, it predicts two main gas accretion episodes. Minor infall episodes are predicted to have followed the second main one but they are of little significance compared to the previous two. By means of this cosmologically motivated infall law, we study the star formation rate, the SNIa and SNII rate, the total amount of gas and stars in the solar neighbourhood and the behaviour of several chemical abundances. We find that the results of the two-infall model are fully compatible with the evolution of the Milky Way with cosmological accretion laws. We derive that the timescale for the formation of the stellar halo and the thick disk must have not been longer than $2 \mathrm{Gyr}$, whereas the disk in the solar vicinity assembled on a much longer timescale ( $\sim 6 \mathrm{Gyr}$ ).

Conclusions. A gas assembly history derived from a DM halo, compatible with the formation of a late-type galaxy from the morphological point of view, can produce chemical properties in agreement with the available observations.
\end{abstract}

Key words. Galaxy: evolution - Galaxy: formation - Galaxy: disk - Galaxy: abundances

\section{Introduction}

In many models of the chemical evolution of the Milky Way, gas infall has been invoked to explain the formation of the Galactic disk (e.g. Chiosi 1980; Matteucci \& François 1989; Lacey \& Fall 1985; Chiappini et al. 1997; Boissier \& Prantzos 2000, among others). Originally, the gas infall was introduced as a possible solution to the G-dwarf problem (Pagel 1989). In general terms the gas infall rate gives the law for the assembly of baryons in a galaxy. However, in the majority of the chemical evolution papers existing in the literature, the gas infall law has been treated as a free parameter with no connection to a galaxy's cosmological context. In other words, in most of the cases the assumed infall law is independent of the details of the galactic dark matter (DM) halo's assembly which, instead, should have a dominant effect on it. On the other hand, the infall law is clearly very important in determining the main characteristics of a galaxy. In this paper we aim at studying the infall law that arises directly from the DM halo and its assembly.

In this way, we will have an infall law for the gas which is related to cosmology and does not contain free parameters. Once achieved, we will test this cosmological infall law in a detailed model of chemical evolution of the Milky Way which follows the evolution of many chemical species by taking into account the stellar lifetimes, detailed nucleosynthesis prescriptions and supernova (type II, Ib/c and Ia) rates. Several authors before us have tried to build a model for the evolution of disk galaxies in a cosmological context, but none of these considered the chemical evolution in such detail as our model. Chemodynamical models for the Milky Way were proposed by Theis et al. (1992), where the evolution of massive spherical galaxies was calculated by a multi-component hydrodynamical approach but with no cosmological context. In Raiteri et al. (1996a,b), instead, $N$-body/hydrodynamical cosmological simulations were used to investigate the chemical evolution of the Galaxy by assuming that it formed by the collapse of a rotating cloud of gas and dark matter. However, their chemical analysis, although detailed, was limited to only oxygen and iron. Another important chemodynamical paper appeared in Samland et al. (1997), in which they presented their two-dimensional chemodynamical code CoDEx. Their model contains nucleosynthesis from supernovae of type I and II and some chemical evolution, but no cosmological context was assumed. More recently, Abadi et al. (2003a,b) presented simulations of galaxy formation in a $\Lambda$ cold dark matter universe $(\Lambda C D M)$ and studied the dynamical and photometric properties of disk galaxies, but no chemical evolution was included.

Robertson et al. (2005) adopted the hierarchical scenario for galaxy formation to see if in this context they could reproduce the rich data set of stellar abundances in the galactic halo and Local Group dwarf galaxies. They used an analytical expression 
for the growth of DM halos in a $\Lambda$ CDM cosmology. Their baryonic infall law is proportional to the DM one. The hierarchical formation scenario, when applied to the stellar halo of the Milky Way, suggests that it formed through accretion and disruption of dwarf galaxies. They concluded that the majority of stars in the stellar halo were formed within a relatively massive dwarf irregular sized dark matter halo, which was accreted and disaggregated $\sim 10 \mathrm{Gyr}$ ago. In their scenario, these systems had rapid star formation histories and were enriched primarily by supernovae ( $\mathrm{SNe}$ ) of type II. They also suggested that the still existing dwarf irregular galaxies formed stars more gradually and they underwent both SNIa and II enrichment. On the other hand, dwarf spheroidal galaxies should be systems where the abundances are determined by galactic winds. In summary, the paper dealt mostly with the comparison between the $[\alpha / \mathrm{Fe}]$ ratios in the galactic halo and dwarf galaxies.

Naab \& Ostriker (2006) studied the metallicity and photometric evolution of a generic disk galaxy by assuming that it forms through mergers of dark matter halos. They took a point of view similar to that of the present paper: in particular, they derived a cosmological infall law and concluded that the infall rate should have been almost constant during the lifetime of the disk. No detailed chemical evolution was followed and no consideration was given to the formation of the stellar halo.

Another paper dealing with chemical evolution in a cosmological context is that by Nagashima \& Okamoto (2006). The authors investigated the chemical evolution in Milky Way-like galaxies based on the CDM model in which cosmic structures form via hierarchical merging. They adopted a semi-analytical model for galaxy formation where the chemical enrichment due to both SNeIa and SNeII was considered. They suggested that the so-called G-dwarf metallicity problem can be fully resolved by the hierarchical formation of galaxies. In fact, the infall term introduced by the traditional monolithic collapse models to solve the G-dwarf problem can be explained by some physical processes such as injection of gas and metals into hot gas due to $\mathrm{SNe}$. The model, however, was not tested on a large number of chemical elements but was limited to the $[\mathrm{O} / \mathrm{Fe}]$ vs. $[\mathrm{Fe} / \mathrm{H}]$ plot.

In this paper we study the effect of different gas infall laws taken from the literature and compare the chemical results with those of François et al.'s (2004) model, which is based on the two-infall model of Chiappini et al. (1997). In the two-infall model it is assumed that the halo and the thick disk formed by means of a first infall episode on a timescale not longer than 2 Gyr, whereas the thin disk should have formed by means of an independent second infall episode lasting much longer. In particular, the timescale for the formation of the solar vicinity was $7 \mathrm{Gyr}$, as suggested by the G-dwarf metallicity distribution, while the internal parts of the thin disk formed faster and the outermost regions are still forming. This scenario has proven to be very successful in reproducing the majority of the properties of the solar vicinity and the whole disk and it was adopted by the majority of the chemical evolution models of the Milky Way. In François et al. (2004) the evolution of 35 chemical species including $\mathrm{C}, \mathrm{N}, \mathrm{O}, \alpha$-elements (Ne, Mg, Si, Ca, Ti), Fe-peak elements plus light elements such as $\mathrm{D}, \mathrm{He}$ and ${ }^{7} \mathrm{Li}$ is followed in detail. In this paper we run a cosmological simulation to find a suitable Dark Matter (DM) halo for a Milky Way-like galaxy by adopting GADGET2 (Springel 2005) and to obtain the infall law for the gas. In particular, we derive the law for the accretion of the DM halo by assuming that the same law is followed by the assembling baryons. Once obtained, this law is tested in the chemical evolution model to see if it is consistent with the two-infall or other scenarios. In order to do that we calculate in detail the evolution of the abundances of several chemical elements, the SN rates and all the physical quantities relevant to the evolution of the solar vicinity. Therefore, we start from a different approach relative to all the previous hierarchical models for the formation of the Milky Way (but see Sommer-Larsen et al. 1999). The reason for considering only gas accretion and not dwarf galaxies, as in other papers, is suggested by the different chemical histories observed in dwarf galaxies relative to the Milky Way (e.g. Lanfranchi \& Matteucci 2004).

The paper is organized as follows: in Sect. 2 we show the nucleosynthesis prescriptions adopted. Section 3 presents a brief description of the model by Chiappini et al. (1997). In Sect. 4 we describe the cosmological simulation, done using the simulator Gadget2. Section 5 describes the adopted infall laws. In Sect. 6 we present the results obtained, comparing the models predictions with the observed properties. Section 7 presents the conclusions.

\section{Nucleosynthesis prescriptions}

One of the most important ingredients for chemical evolution models is represented by the nucleosynthesis prescriptions and consequently by the stellar yields.

The single stars in the mass range $0.8 M_{\odot} \leq M \leq 8 M_{\odot}$ (low and intermediate-mass stars) contribute to the Galactic enrichment through planetary nebula ejection and quiescent mass loss. They enrich the interstellar medium mainly in $\mathrm{He}, \mathrm{C}, \mathrm{N}$ and heavy s-process elements (e.g. Cescutti et al. 2006). We adopt here the stellar yields for low and intermediate mass stars of van den Hoek \& Groenewegen (1997) computed as functions of stellar metallicity, their case with variable mass loss. These stars are also the progenitor of type Ia supernovae ( $\mathrm{SNe}$ ), if they are in binary systems that originate from carbon deflagration of $\mathrm{C}-\mathrm{O}$ white dwarfs. We adopt in this paper the single-degenerate progenitor scenario (Whelan \& Iben 1973; Han \& Podsiadlowski 2004). Type Ia SNe contribute a substantial amount of $\mathrm{Fe}\left(\sim 0.6 M_{\odot}\right.$ per event $)$ and Fe-peak elements as well as non negligible quantities of $\mathrm{Si}$ and $\mathrm{S}$. They also produce other elements, such as $\mathrm{O}, \mathrm{C}, \mathrm{Ne}, \mathrm{Ca}, \mathrm{Mg}$ and $\mathrm{Ni}$, but in very small amounts compared to type II SNe. We assume the stellar yields for type Ia SNe from Iwamoto et al. (1999).

Massive stars $\left(8 M_{\odot}<M \leq 100 M_{\odot}\right)$ are the progenitor of core-collapse $\mathrm{SNe}$ which can be either type II SNe or type $\mathrm{Ib} / \mathrm{c} \mathrm{SNe}$. These latter can arise from binary systems or Wolf-Rayet stars whereas type II SNe originate from the massive stars in the lower mass range. Type II SNe mainly produce the so called $\alpha$-elements, such as $\mathrm{O}, \mathrm{Mg}, \mathrm{Ne}, \mathrm{Ca}, \mathrm{S}$ and $\mathrm{Si}$ and $\mathrm{Ti}$, but also some $\mathrm{Fe}$ and $\mathrm{Fe}$-peak elements although in smaller amounts than type Ia SNe. We adopt here the stellar yields for massive stars by Woosley \& Weaver (1995) with the suggested modifications of François et al. (2004). However, the most important modifications concern some Fe-peak elements, except Fe itself, whereas for the $\alpha$-elements, with the exception of $\mathrm{Mg}$ which has been increased relative to the original yields, the yields are substantially unmodified. The modifications of the yields in François et al. (2004) were required to fit at best and at the same time the $[$ element/Fe] versus $[\mathrm{Fe} / \mathrm{H}]$ patterns and the solar absolute abundances. We keep the same prescriptions here with the purpose of testing the infall laws without changing the other model parameters.

We start with primordial gas and the assumed primordial abundances of D and ${ }^{3} \mathrm{He}$ we have chosen: $3.90 \times 10^{-5}$ and $2.25 \times 10^{-5}$, respectively. The reference solar abundances are those by Asplund et al. (2005). 


\section{The model by Chiappini et al. (1997)}

Prior to the two-infall model of Chiappini et al. (1997), different models assuming gas accretion onto the galactic disk had been constructed. For example, dynamical models, such as the one of Larson (1976), viscous models (Lacey \& Fall 1985; Sommer-Larsen \& Yoshii 1989, 1990; Tsujimoto et al. 1995), inhomogeneous models (Malinie et al. 1993), detailed chemical evolution models (Matteucci \& Greggio 1986; Tosi 1988; Matteucci \& François 1989; Pagel 1989; Matteucci \& François 1992; Carigi 1994; Giovagnoli \& Tosi 1995; Ferrini et al. 1994; Pardi \& Ferrini 1994; Pardi et al. 1995; Prantzos \& Aubert 1995; Timmes et al. 1995) and chemodynamic models (Samland \& Hensler 1996; Burkert et al. 1992). The model by Chiappini et al. (1997) was the first in which two main infall episodes for the formation of the Galactic components were suggested. In particular, they assumed that the first infall episode was responsible for the formation of the halo and thick-disk stars that originated from a fast dissipative collapse. The second infall episode formed the thin-disk component, with a timescale much longer than that of the thick-disk formation. The authors also included in the model a threshold in the gas density below which the star formation process stops. The existence of such a threshold value is suggested by observations relative to the star formation in external disk galaxies (Kennicutt 1998; but see Boissier et al. 2006). The physical reason for a threshold in the star formation is related to the gravitational stability, according to which, below a critical density, the gas is stable against density condensations and, consequently, the star formation is suppressed. In the two-infall model the halo-thick disk and the thin disk evolutions occur at different rates, mostly as a result of different accretion rates. With these precise prescriptions it is possible to reproduce the majority of the observed properties of the Milky Way and this shows how important the choice is of the accretion law for the gas coupled with the star formation rate in the Galaxy evolution.

In the model by Chiappini et al. (1997) the Galactic disk is approximated by a series of concentric annuli, $2 \mathrm{kpc}$ wide, without exchange of matter between them. The basic equations are the same as in Matteucci \& François (1989). The two main differences between the model by Chiappini et al. (1997) and Matteucci \& François (1989) are the rate of mass accretion and the rate of star formation. Moreover, in the model by Chiappini et al. (1997) the material accreted by the Galactic thin disk comes mainly from extragalactic sources. These extragalactic sources could include, for instance, the Magellanic Stream or a major accretion episode (see Beers \& Sommer-Larsen 1995, and references therein). The two models have in common the "inside-out" formation of the thin disk, in the sense that both assume that the timescale for the disk formation increase with galactocentric distance (see Sect. 5). This choice was dictated by the necessity of reproducing the abundance gradients along the Galactic disk.

The SFR is a Schmidt (1955) law with a dependence on the surface gas density ( $k=1.5$, see Kennicutt 1998) and also on the total surface mass density (see Dopita \& Ryder 1994). In particular, the SFR is based on the law originally suggested by Talbot \& Arnett (1975) and then adopted by Chiosi (1980):

$\psi(r, t)=v\left(\frac{\Sigma(r, t) \Sigma_{\mathrm{gas}}(r, t)}{\Sigma\left(r_{\odot}, t\right)^{2}}\right)^{(k-1)} \Sigma_{\mathrm{gas}}(r, t)^{k}$,

where the constant $v$ is the efficiency of the star formation process and is expressed in $\mathrm{Gyr}^{-1}$ : in particular, $v=2 \mathrm{Gyr}^{-1}$ for the halo and $1 \mathrm{Gyr}^{-1}$ for the disk ( $\left.t \geq 1 \mathrm{Gyr}\right)$. The total surface mass density is represented by $\Sigma(r, t), \Sigma\left(r_{\odot}, t\right)$ is the total surface mass density at the solar position, assumed to be $r_{\odot}=8 \mathrm{kpc}$ (Reid 1993). The quantity $\Sigma_{\text {gas }}(r, t)$ represents the surface gas density and $t$ represents the time. These choices of values for the parameters allow the model to fit the observational constraints very well, in particular in the solar vicinity. A threshold gas density for the star formation in the disk of $7 M_{\odot} \mathrm{pc}^{-2}$ is adopted in all the models presented here.

The IMF is that of Scalo (1986) normalized over a mass range of $0.1-100 M_{\odot}$ and it is assumed to be constant in space and time.

\section{The cosmological simulation}

The main aim of our work is to follow the chemical evolution of spiral galaxies in a cosmological context. To this aim, we run a dark matter-only cosmological simulation, using the public treecode GADGET2 (Springel 2005), in order to produce and study dark matter halos in which spiral galaxies can form. Our simulated box has a side of $24 h^{-1} \mathrm{Mpc}$. We used $256^{3}$ particles. We adopted the standard cosmological parameters from WMAP 3 -years (Spergel et al. 2007), namely $\Omega_{0}=0.275, \Omega_{\lambda}=0.725$ and $\Omega_{b}=0.041$. Each DM particle has a mass equal to $6.289 \times$ $10^{7} h^{-1} M_{\odot}$ and the Plummer-equivalent softening length is set to $3.75 h^{-1}$ comoving kpc to redshift $z=2$ and to $1.25 h^{-1}$ physical kpc since $z=2$. We use the public package GRAFIC (Bertschinger 1995) to set up our initial conditions. The simulation started at redshift $z=20$ and 28 outputs were produced. We have chosen to use a quite large spread in the redshifts at the beginning, while in the last part of the simulation, where a small change in the redshift corresponds to a large change in time, the redshifts are closer. We checked that the final mass function of DM halos and the power spectrum are in agreement with theoretical expectations.

We identified DM halos at redshift $z=0$ using a standard friend-of-friends algorithm, with a linking length $l=0.17$ mean (comoving) interparticle distance. After that, we determined the virial mass and radius for each DM halo, using the center of mass of the F-o-F group as the halo center. Here we define the virial radius as the radius of the sphere within which the matter density contrast is $\delta \approx 100$ times the critical density, with $\delta$ given by the cosmological parameter as in Navarro \& Steinmetz (2000).

We then built the mass accretion history of our halos. To achieve this goal, we analysed 28 outputs from redshift $z=9.0$ to $z=0$. We identified all DM halos in each snapshot using the procedure described above, except for the fact that we used the redshift-dependent density contrast given by Bryan \& Norman (1997) to define the virial radius as a function of $z$. At any output $z_{i+1}$, we found all the progenitors of our halos at redshift $z_{i}$. We defined a halo at redshift $z_{i+1}$ to be a progenitor of one at $z_{i}$ if at least $50 \%$ of its particles belong to the candidate offspring (see e.g. Kauffmann 2001; Springel et al. 2001, for a discussion of this threshold). The mass accretion history is defined as the mass of the main progenitor of the halo as a function of redshift. Having the mass accretion histories, we were able to identify the redshift of formation (defined as the epoch at which half of the mass of the forming halos were accreted) and the redshift at which each halo experienced its last major merger (defined as an increase of at least $25 \%$ of its mass with respect to the mass of its main progenitor at the previous redshift). To identify the DM halos that can host a spiral galaxy similar to the MW we used selection criteria based on four different characteristics of the halos:

- mass between $5 \times 10^{11} M_{\odot}$ and $5 \times 10^{12} M_{\odot}$; 
Table 1. Characteristics of the chosen DM halos.

\begin{tabular}{ccccc}
\hline \hline Group & Mass $\left[10^{10} M_{\odot}\right]$ & Spin parameter & Redshift major merger & Redshift of formation \\
\hline 48001 & 90.26 & 0.045 & 5.00 & $1.75-1.50$ \\
52888 & 465.75 & 0.059 & 3.75 & $1.50-1.25$ \\
56009 & 90.73 & 0.049 & 3.25 & $2.00-1.75$ \\
6460 & 61.94 & 0.041 & 2.50 & $1.25-1.00$ \\
\hline
\end{tabular}

- spin parameter $\lambda>0.04$

- redshift of last major merger larger than $z=2.5$;

- redshift of formation larger than $z=1.0$.

We found four DM halos compatible with our selection criteria. We label them with their F-o-F group number, i.e. group 48001 , group 52888, group 56004 and group 6460. We note that, given our simulated volume, the expected number of halos in our mass range is higher: using a Press \& Schechter mass function, approximately 70 halos are expected. However, the requirement of having a "quiescent" formation history and a high spin parameter greatly reduces their number (see e.g. D'Onghia \& Burkert 2004, and references therein for a discussion on this point). In this paper we want to focus on the chemical evolution of a MWlike galaxy in its cosmological context, and therefore we will not discuss issues connected with the angular momentum problem which arises when performing a direct simulation of the formation of a disk-like galaxy in a cosmological dark matter halo. Also, we could have obtained a larger number of halos by relaxing the third of the above constraints, but for the purpose of the present work it is more important to focus on the most promising DM halos than on obtaining statistics. So, we simply used the (few) best candidates as example halos.

Assuming that the baryonic matter follows the same accretion pattern as the dark matter, and that it represents $19 \%$ (the cosmological baryon fraction) of all the infalling matter, we obtained a final baryonic mass for the Galaxy of $1.7 \times 10^{11} M_{\odot}$. This approach is similar to that followed by Robertson et al. (2005) except that we did not make any hypothesis on the fraction of cold gas falling into the disk but we used the observations to fix it. In this way, we obtained the baryon infall law from the mass accretion history of each halo.

Here, we do not make any attempt to model the disk formation inside the hierarchically growing DM halo. This is undoubtedly an over-simplification of the physics involved. On the other hand, the issue of disk galaxy formation in hierarchical cosmologies is far from being solved. Any attempt to model the formation of the disk should use a number of assumptions which are currently under debate. As an example, the structure of the disk is obviously driven by the gas cooling coupled with its angular momentum content. Semi-analytical galaxy formation models (SAMs) usually assume that DM and gas share the same specific angular momentum. But this point is very controversial (see e.g. D'Onghia \& Burkert 2004; D'Onghia et al. 2006, and references therein). Even direct self consistent numerical simulations are not currently able to solve the problem, which may (Governato et al. 2007) or may not (Abadi et al. 2003a,b) be simply due to insufficient numerical resolution and/or an insufficiently detailed treatment of supernova feedback. Lacking a widely accepted model for the formation of the disk, we prefer to keep our model as simple as possible and to verify whether the cosmological growth of the halo is compatible with the observational constraints obtained using available data on the chemical composition of stars and gas in the Milky Way.

In particular, we assumed that the derived infall law has the same functional form for the whole Milky Way, but that the
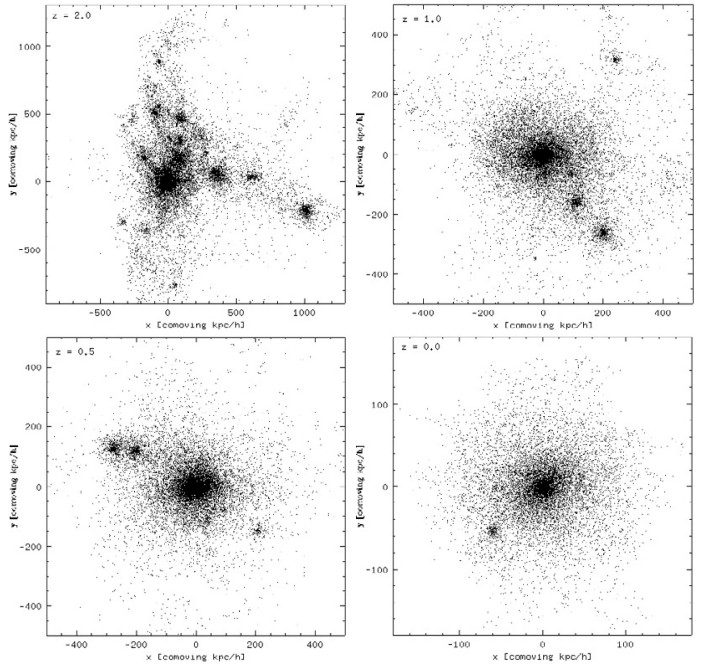

Fig. 1. This figure represents our best cosmological halo, i.e. halo 48001, at four different redshifts $(z=2.0, z=1.0, z=0.5$ and $z=0.0)$.

normalization constant is different for different Galactic regions. In other words, the normalization constants were obtained by reproducing the current total surface mass density at any specific galactocentric distance (see next section), although here we will focus on the solar neighbourhood, leaving to a forthcoming paper a more detailed study of the whole Galactic disk. Finally, we also considered the arithmetic mean of the infall laws of all four halos, in order to have an "average" cosmological infall law to study. In Table 1 we summarize the characteristics of the halos. Figure 1 represents our best cosmological halo (halo 48001) at four different redshifts $(z=0.0, z=0.5, z=1.0$ and $z=2.0)$.

\section{The infall laws}

In testing the accretion laws, we started by adopting the twoinfall law model, as suggested by Chiappini et al. (1997). This law presents two distinct peaks. During the first peak the halo and thick disk formed whereas during the second peak the thin disk was assembled. The two accretion events are considered to be independent from each other and only a very small fraction of the gas lost from the halo was assumed to have fallen onto the disk. The infall law that we indicate as $A(r, t)$ is expressed as:

$$
A(r, t)=a(r) \mathrm{e}^{-t / \tau_{\mathrm{H}}(r)}+b(r) \mathrm{e}^{-\left(t-t_{\max }\right) / \tau_{\mathrm{D}}(r)}\left[M_{\odot} \mathrm{pc}^{-2} \mathrm{Gyr}^{-1}\right],
$$

where $a(r)$ and $b(r)$ are two parameters fixed by reproducing the total present time surface mass density along the Galactic disk. In particular, in the solar vicinity the total surface mass density $\Sigma_{\text {tot }}=51 \pm 6 M_{\odot} \mathrm{pc}^{-2}$ (see Boissier \& Prantzos 1999). $t_{\max }=1.0 \mathrm{Gyr}$ is the time for the maximum infall on the thin disk, $\tau_{\mathrm{H}}=2.0 \mathrm{Gyr}$ is the time scale for the formation of the halo thick-disk and $\tau(r)$ is the timescale for the formation of the thin disk and is a function of the galactocentric distance 


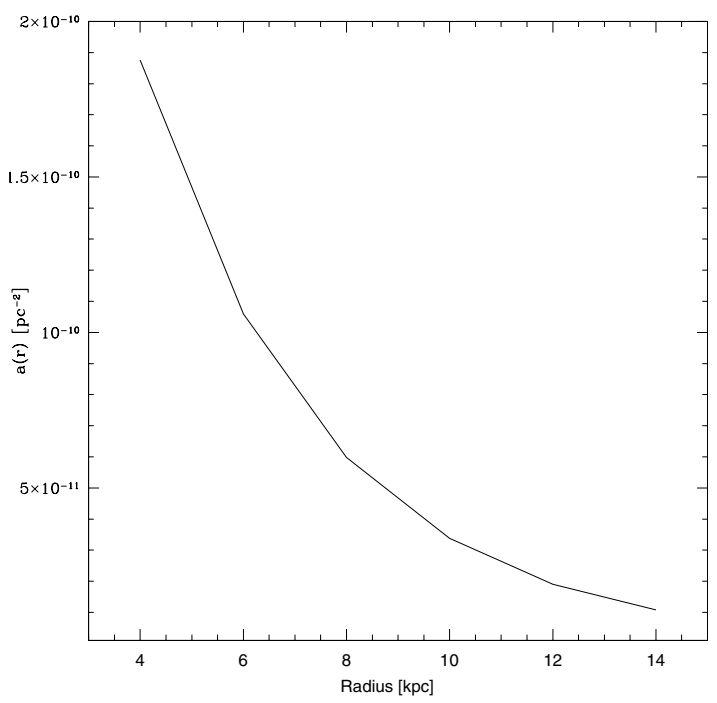

Fig. 2. $a(r)$ vs. radius. This normalization constant is fixed to reproduce the present time total surface mass density along the disk (see Eq. (6)).

(inside-out formation, Matteucci \& François 1989; Chiappini et al. 2001). In particular, it is assumed that:

$\tau_{\mathrm{D}}=1.033 r(\mathrm{kpc})-1.267[\mathrm{Gyr}]$.

Besides this infall law, we tested other possible laws, such as a time constant infall rate. In particular:

$A(r, t)=3.80\left[M_{\odot} \mathrm{pc}^{-2} \mathrm{Gyr}^{-1}\right]$.

This law is probably not realistic although Naab \& Ostriker (2006) concluded that an almost constant infall law over the disk lifetime was to be preferred. Here we adopted it mainly for the purpose of comparison with more realistic laws. We adopted that particular value of the infall rate in order to reproduce the current infall rate (see Table 3), as well as the current total surface mass density.

The third infall law we tested is a linear infall law, given by:

$A(r, t)=6.57-0.4 \cdot t\left[M_{\odot} \mathrm{pc}^{-2} \mathrm{Gyr}^{-1}\right]$.

Again, we used this particular expression in order to reproduce the current $\Sigma_{\text {tot }}$ and infall rate.

The fourth adopted infall law is the same as that of Chiappini et al. (1997) but with pre-enriched infalling gas. The metallicity of the infalling gas which forms the disk was assumed to be 10 times lower than the present time interstellar medium (ISM) metallicity while the infalling gas which forms the halo is still primordial. The assumed chemical composition of the infalling gas does not assume solar abundance ratios but reflects the composition of the halo-thick disk.

Then, we tested the infall laws derived from the cosmological simulations performed with GADGET2 (Springel 2005), as described before. In particular, to derive the cosmological infall law we proceeded in the following way:

$A(r, t)=a(r) 0.19 \frac{\mathrm{d} M_{\mathrm{DM}}}{\mathrm{d} t}\left[M_{\odot} \mathrm{pc}^{-2} \mathrm{Gyr}^{-1}\right]$.

where 0.19 is the cosmological baryonic fraction and $a(r)$ is a normalization constant fixed to reproduce the current total surface mass density along the disk, in analogy with Eq. (2). For the solar ring $a(r)=\frac{\Sigma\left(r_{\odot}, t_{\mathrm{G}}\right)}{M_{\mathrm{Gal}}}$, with $M_{\mathrm{Gal}}=0.19 M_{\mathrm{DM}}$ being the baryonic mass of our Galaxy and $t_{\mathrm{G}}$ the Galactic lifetime. In Fig. 2 we show the values of $a(r)$ versus the galactocentric distance.
Table 2. Model parameters. In the first column is the number of the model, in the second one the adopted infall law, in the third the time scale for the halo, in the fourth that for the disk and in the fifth the type of infalling gas. All the models adopt a threshold gas density for star formation in the disk of $7 M_{\odot} \mathrm{pc}^{-2}$. The model by Naab \& Ostriker (2006) is the only one that has a threshold also during the formation of the halo. Note that our best cosmological model is Model 5.

\begin{tabular}{ccccc}
\hline \hline Model & $\begin{array}{c}\text { Infall law } \\
{\left[M_{\odot} \mathrm{pc}^{-2} \mathrm{Gyr}^{-1}\right]}\end{array}$ & $\begin{array}{c}\tau \text { halo } \\
{[\mathrm{Gyr}]}\end{array}$ & $\begin{array}{c}\tau \text { disk } \\
{[\mathrm{Gyr}]}\end{array}$ & Gas $t=0$ \\
\hline 1 & Two-infall law & 0.8 & 7 & Primordial \\
2 & 3.80 & 0.8 & 7 & Primordial \\
3 & $6.57-0.40 \cdot T$ & 0.8 & 7 & Primordial \\
4 & Two-infall law & 0.8 & 7 & Enriched $\left(1 / 10 Z_{\text {today }}\right)$ \\
5 & Group 48001 & - & - & Primordial \\
6 & Group 52888 & - & - & Primordial \\
7 & Group 56009 & - & - & Primordial \\
8 & Group 6460 & - & - & Primordial \\
9 & Mean & - & - & Primordial \\
10 & Naab \& Ostriker & - & - & Primordial \\
\hline
\end{tabular}

One infall law is given by the arithmetic average of the infall laws derived for the four halos and the last infall law is that suggested by Naab \& Ostriker (2006). In Table 2 we show the model parameters. The different models are identified mainly by their infall histories.

Our infall laws for the solar region $(8 \mathrm{kpc}$ from the Galactic center) are shown in Fig. 3; in Fig. 4 we show the increase in time of the total surface mass density obtained by the mass accretion history of the simulated halos. The infall law derived for the best halo selected as representative of the Milky Way halo is very similar to the two-infall law by Chiappini et al. (1997).

We selected our best halo by choosing the one which has a very high redshift of the last major merger. This is to ensure the correct spin parameter for a Milky Way-like galaxy. The assembly history of this particular halo presents two distinct accretion peaks which produce an infall law very similar to the two-infall model by Chiappini et al. (1997). The only difference to the twoinfall model is that in this case the two peaks are placed at a lower redshifts. After the two main peaks there are other smaller peaks. All models predict a current infall rate which is in good agreement with the observed one, as quoted by Naab \& Ostriker (2006). Thus, according to the infall laws derived from cosmological simulations, the Galaxy had some large infall episodes at high redshift, followed by smaller ones.

In Fig. 4 we present the total surface mass density $\Sigma_{\text {tot }}$, expressed as $M_{\odot} \mathrm{pc}^{-2}$, as a function of time for all the models. Once again Models 1 and 4 (two-infall model with primordial and enriched infall, respectively) have the same $\Sigma_{\text {tot }}$. The linear model predicts the largest final amount of matter, equal to $51.88 M_{\odot} \mathrm{pc}^{-2}$. The constant model has a linear growth (in this case $\Sigma_{\text {tot }}$ is the integral of a constant infall law) and produces $49.98 M_{\odot} \mathrm{pc}^{-2}$. Model 10, i.e. the model of Naab \& Ostriker (2006), is the only one that starts to increase the amount of matter very slowly (in the solar neighbourhood). After $5 \mathrm{Gyr}$ from the Big Bang it only reached $6.00 M_{\odot} \mathrm{pc}^{-2}$. The cosmological models produce results that are quite similar to the twoinfall model. At the beginning their growth is slower but after $\sim 3-3.5$ Gyr their $\Sigma_{\text {tot }}$ increases with a steeper slope, due to the peaks in the infall law.

In Fig. 5 we show the infall law derived from Model 5 (our best halo) for three galactocentric distances (4, 8 and $14 \mathrm{kpc}$ ). As one can see the accretion histories are different at different galactocentric distances, although no assumptions are used about 


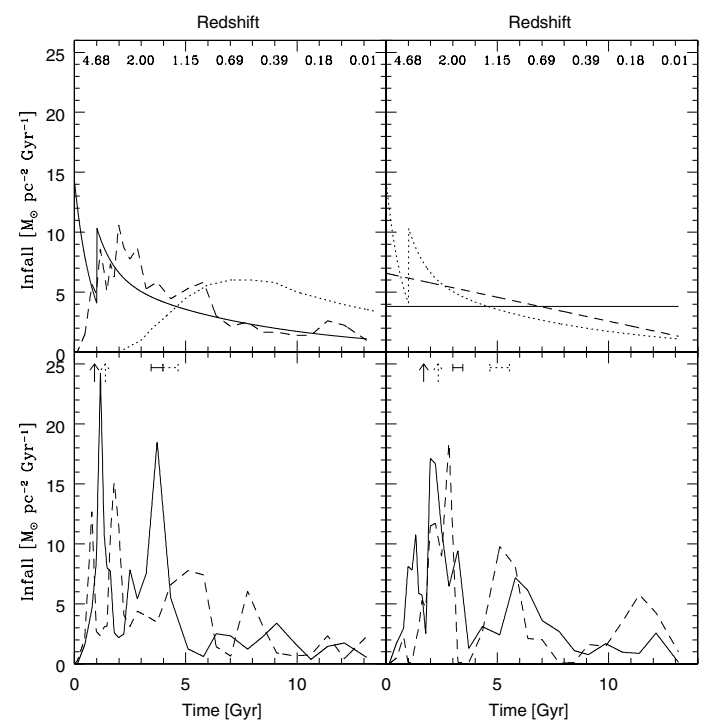

Fig. 3. Infall vs. time. Upper left panel: red solid line is the two-infall model (Model 1); black dashed line is the cosmological mean model (Model 9); green dotted line is the model by Naab \& Ostriker (2006) (Model 10). Upper right panel: magenta solid line is the constant infall model (Model 2); blue dashed line is the linear infall model (Model 3); cyan dotted line is the pre-enriched model $\left(Z_{\text {inf }}=1 / 10 Z_{\text {today }}\right.$, Model 4$)$. Bottom left panel: black solid line is Model 5; magenta dashed line is Model 6. Bottom right panel: blue solid line is Model 7; cyan dashed line is Model 8. In the bottom left panel the black solid arrow represents the redshift of last major merger for Model 5, the magenta dotted arrow the redshift of last major merger for Model 6, the black solid interval the redshift of formation for Model 5 and the magenta dotted interval the redshift of formation for Model 6. In the bottom right panel the blue solid arrow represents the redshift of last major merger for Model 7, the cyan dotted arrow the redshift of last major merger for Model 8, the blue solid interval the redshift of formation for Model 7 and the cyan dotted interval the redshift of formation for Model 8.

the timescales of disk formation at any radius. This particular behaviour of the infall law with radius needs to be tested and this will be the subject of a forthcoming paper. We have only checked the gradient of $\mathrm{O}$ along the galactic disk which is predicted to be very similar to the one obtained with the two-infall model for $R_{\mathrm{G}}>8 \mathrm{kpc}$, whereas it is flatter for $R_{\mathrm{G}} \leq 8 \mathrm{kpc}$ (see Sect. 6). Clearly the formation of the bulge is included in the accretion history of the first $2 \mathrm{kpc}$.

\section{Results}

In this section we present the chemical evolution results. Results are shown in Tables 3-5. In Table 3 we show the predicted current star formation rates, the current infall and the current SNIa and SNII rates, compared with the corresponding observational values. In Table 4 we plot the total amount of gas and the number of stars, the $\Sigma_{\text {gas }} / \Sigma_{\text {tot }}$ and the total surface mass density in a ring of $2 \mathrm{kpc}$ centered at the Sun's galactocentric distance $(8 \mathrm{kpc})$. Table 5 presents the predicted solar absolute abundances by mass for $\mathrm{Fe}, \mathrm{C}, \mathrm{Mg}, \mathrm{N}, \mathrm{O}$ and $\mathrm{Si}$, namely the abundances in the ISM at the time of birth of the solar system $4.5 \mathrm{Gyr}$ ago, compared with the observed ones by Asplund et al. (2005).

Figure 6 shows the star formation rate as a function of cosmic time for all the models. At high redshift there is a gap in the SFR for some of the models. This gap is due to the adoption of a threshold in the surface gas density below which star formation does not occur. In all models we have adopted a threshold equal

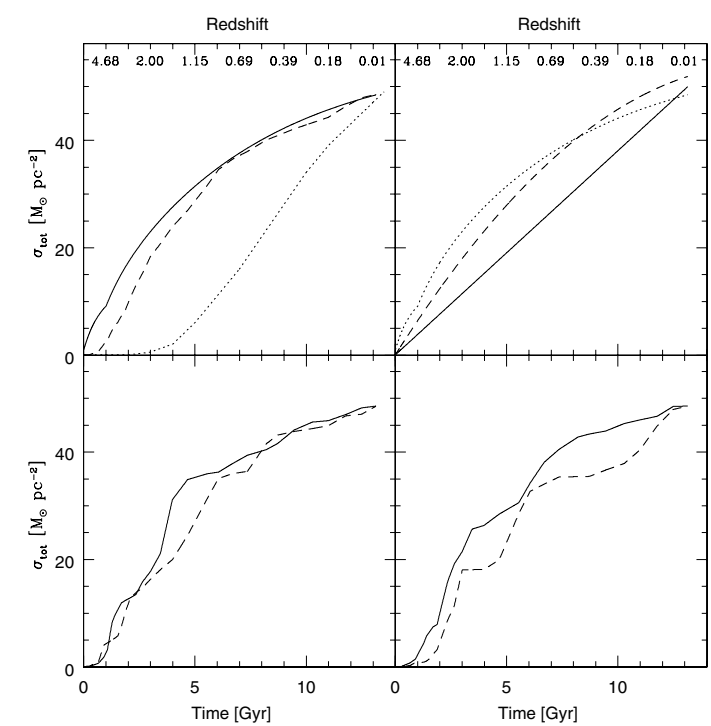

Fig. 4. $\Sigma_{\text {tot }}$ vs. time. Upper left panel: red solid line is the two-infall model (Model 1); black dashed line is the cosmological mean model (Model 9); green dotted line is the model by Naab \& Ostriker (2006) (Model 10). Upper right panel: magenta solid line is the constant infall model (Model 2); blue dashed line is the linear infall model (Model 3); cyan dotted line is the pre-enriched model $\left(Z_{\text {inf }}=1 / 10 Z_{\text {today }}\right.$, Model 4$)$. Bottom left panel: black solid line is Model 5; magenta dashed line is Model 6. Bottom right panel: blue solid line is Model 7; cyan dashed line is Model 8.

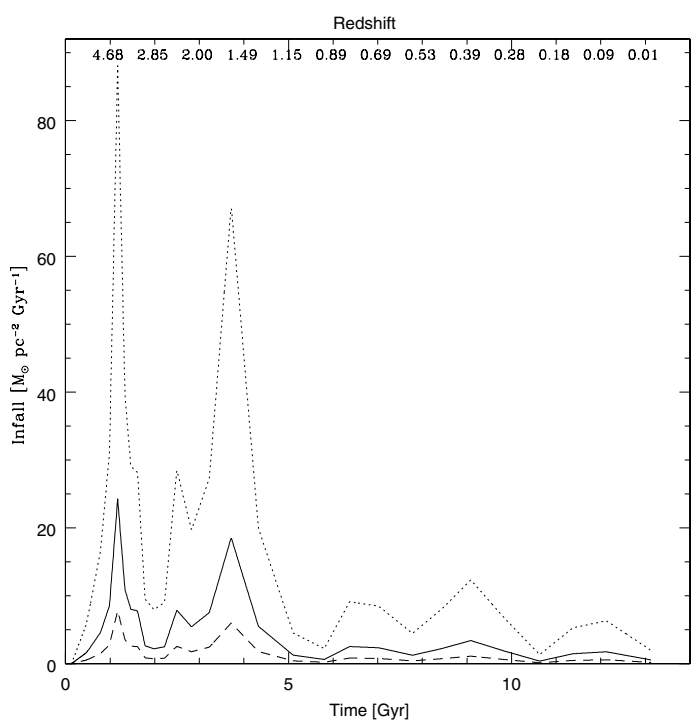

Fig. 5. The infall law of our best cosmological halo, i.e. halo 48001 , at three different radii (4 kpc: blue dotted line; $8 \mathrm{kpc}$ : red solid line; $14 \mathrm{kpc}$ : green dashed line).

to $7.0 M_{\odot} \mathrm{pc}^{-2}$ during the formation of the thin disk. Model 10 instead, adopting the infall law suggested by Naab \& Ostriker (2006), has a star formation threshold equal to $7.0 M_{\odot} \mathrm{pc}^{-2}$ both for the halo and the disk.

From Fig. 6 we deduce that the constant infall model predicts a growing star formation rate at low redshifts, a trend that is not predicted by the other laws. On the other hand, the cosmological best model (Model 5) predicts a very important peak between 3 and 6 Gyr, which should correspond to the formation of the bulk of the stars in the thin disk. This peak is directly related to the trend of the infall law. After 10 Gyr from the Big Bang the 
Table 3. Current values for all the models and observed values as reported in Boissier \& Prantzos (1999) and Chiappini et al. (2001).

\begin{tabular}{ccccc}
\hline \hline Model & $\begin{array}{c}\text { SFR } \\
{\left[M_{\odot} \mathrm{pc}^{-2} \mathrm{Gyr}^{-1}\right]}\end{array}$ & $\begin{array}{c}\text { Infall } \\
{\left[M_{\odot} \mathrm{pc}^{-2} \mathrm{Gyr}^{-1}\right]}\end{array}$ & $\begin{array}{c}\text { SNII rate } \\
{\left[\mathrm{pc}^{-2} \mathrm{Gyr}^{-1}\right]}\end{array}$ & $\begin{array}{c}\text { SNIa rate } \\
{\left[\mathrm{pc}^{-2} \mathrm{Gyr}^{-1}\right]}\end{array}$ \\
\hline 1 & 2.66 & 1.100 & 0.00900 & 0.00330 \\
2 & 4.55 & 3.800 & 0.01928 & 0.00411 \\
3 & 2.81 & 1.320 & 0.01194 & 0.00391 \\
4 & 2.66 & 1.100 & 0.00900 & 0.00332 \\
5 & 2.65 & 0.528 & 0.00584 & 0.00366 \\
6 & 2.69 & 2.273 & 0.01140 & 0.00347 \\
7 & 2.65 & 0.126 & 0.00229 & 0.00366 \\
8 & 4.01 & 0.998 & 0.01712 & 0.00412 \\
9 & 2.69 & 0.979 & 0.01147 & 0.00381 \\
10 & 4.72 & 3.406 & 0.02002 & 0.00397 \\
\hline Boissier \& Prantzos (1999) & $2-5$ & $1.0-3.3$ & 0.02 & $0.0042 \pm 0.0016$ \\
\hline Chiappini et al. (2001) & 2.6 & 1.0 & 0.008 & 0.004 \\
\hline
\end{tabular}

Table 4. Current values for all the models and observed values as reported in Boissier \& Prantzos (1999) and Chiappini et al. (2001).

\begin{tabular}{ccccc}
\hline \hline Model & $\begin{array}{c}\text { Gas } \\
{\left[M_{\odot} \mathrm{pc}^{-2}\right]}\end{array}$ & $\begin{array}{c}\text { Stars } \\
{\left[M_{\odot} \mathrm{pc}^{-2}\right]}\end{array}$ & $\begin{array}{c}\Sigma_{\text {gas }} \\
\Sigma_{\text {tot }}\end{array}$ & $\begin{array}{c}\text { Total } \\
{\left[M_{\odot} \mathrm{pc}^{-2}\right]}\end{array}$ \\
\hline 1 & 7.00 & 35.24 & 0.1444 & 48.46 \\
2 & 10.11 & 35.09 & 0.2024 & 49.98 \\
3 & 7.42 & 38.66 & 0.1431 & 51.88 \\
4 & 7.00 & 35.24 & 0.1444 & 48.46 \\
5 & 6.99 & 36.13 & 0.1439 & 48.55 \\
6 & 7.06 & 35.60 & 0.1455 & 48.53 \\
7 & 7.00 & 36.69 & 0.1442 & 48.56 \\
8 & 9.21 & 34.75 & 0.2056 & 48.55 \\
9 & 7.06 & 36.32 & 0.1455 & 48.55 \\
10 & 10.29 & 34.29 & 0.2099 & 49.04 \\
\hline Boissier \& Prantzos (1999) & $13 \pm 3$ & $35 \pm 5$ & $0.15-0.25$ & $51 \pm 6$ \\
\hline Chiappini et al. (2001) & 7.0 & 36.3 & 0.13 & 53.85 \\
\hline
\end{tabular}

Table 5. Predicted and observed solar abundances by mass (after 8.64 Gyr from the Big Bang).

\begin{tabular}{ccccccc}
\hline \hline Model & $\mathrm{Fe}$ & $\mathrm{C}$ & $\mathrm{Mg}$ & $\mathrm{N}$ & $\mathrm{O}$ & $\mathrm{Si}$ \\
\hline 1 & $0.162 \mathrm{E}-02$ & $0.156 \mathrm{E}-02$ & $0.774 \mathrm{E}-03$ & $0.121 \mathrm{E}-02$ & $0.592 \mathrm{E}-02$ & $0.980 \mathrm{E}-03$ \\
2 & $0.987 \mathrm{E}-03$ & $0.119 \mathrm{E}-02$ & $0.585 \mathrm{E}-03$ & $0.932 \mathrm{E}-03$ & $0.461 \mathrm{E}-02$ & $0.665 \mathrm{E}-03$ \\
3 & $0.149 \mathrm{E}-02$ & $0.135 \mathrm{E}-02$ & $0.778 \mathrm{E}-03$ & $0.105 \mathrm{E}-02$ & $0.602 \mathrm{E}-02$ & $0.940 \mathrm{E}-03$ \\
4 & $0.111 \mathrm{E}-02$ & $0.157 \mathrm{E}-02$ & $0.691 \mathrm{E}-03$ & $0.121 \mathrm{E}-02$ & $0.547 \mathrm{E}-02$ & $0.771 \mathrm{E}-03$ \\
5 & $0.169 \mathrm{E}-02$ & $0.199 \mathrm{E}-02$ & $0.797 \mathrm{E}-03$ & $0.142 \mathrm{E}-02$ & $0.608 \mathrm{E}-02$ & $0.102 \mathrm{E}-02$ \\
6 & $0.917 \mathrm{E}-03$ & $0.140 \mathrm{E}-02$ & $0.604 \mathrm{E}-03$ & $0.105 \mathrm{E}-02$ & $0.483 \mathrm{E}-02$ & $0.653 \mathrm{E}-03$ \\
7 & $0.107 \mathrm{E}-02$ & $0.168 \mathrm{E}-02$ & $0.701 \mathrm{E}-03$ & $0.124 \mathrm{E}-02$ & $0.559 \mathrm{E}-02$ & $0.761 \mathrm{E}-03$ \\
8 & $0.126 \mathrm{E}-02$ & $0.212 \mathrm{E}-02$ & $0.796 \mathrm{E}-03$ & $0.144 \mathrm{E}-02$ & $0.635 \mathrm{E}-02$ & $0.879 \mathrm{E}-03$ \\
9 & $0.111 \mathrm{E}-02$ & $0.173 \mathrm{E}-02$ & $0.716 \mathrm{E}-03$ & $0.127 \mathrm{E}-02$ & $0.570 \mathrm{E}-02$ & $0.783 \mathrm{E}-03$ \\
10 & $0.531 \mathrm{E}-03$ & $0.102 \mathrm{E}-02$ & $0.439 \mathrm{E}-03$ & $0.784 \mathrm{E}-03$ & $0.362 \mathrm{E}-02$ & $0.432 \mathrm{E}-03$ \\
\hline Asplund et al. (2005) & $0.116 \mathrm{E}-02$ & $0.217 \mathrm{E}-02$ & $0.601 \mathrm{E}-03$ & $0.623 \mathrm{E}-03$ & $0.540 \mathrm{E}-02$ & $0.669 \mathrm{E}-03$ \\
\hline
\end{tabular}

threshold is easily reached in most of the models, thus causing the SFR to have an oscillating behaviour.

In Fig. 7 we present the SNIa rates for all the models. The cosmological law of Model 5 predicts a peak for the SNIa rate at about 6 Gyr. This is due to the fact that the SFR in this model has a large peak at about 5 Gyr. Thanks to this peak, many stars form and many SNIa explode after a delay of about 1 Gyr. All the models predict a SNIa rate between 0.003 and $0.004 \mathrm{SNe} \mathrm{pc}^{-2} \mathrm{Gyr}^{-1}$, in good agreement with the value given by Boissier \& Prantzos (1999), i.e. $0.0042 \pm 0.0016$.

We do not show the rates of SNe II since their behaviour is like that of the SFR. Type II SNe are produced by massive stars that live only a few million years. For this reason, the behaviour of the SNII rate is equivalent to that of the SFR.

In Fig. 8 we present the predicted $[\mathrm{Fe} / \mathrm{H}]$ as a function of time for all models. The model with a constant infall law (Model 2) and Model 10 never reach the solar abundance. The reason is that in both models the infall rate during the whole galactic lifetime is probably overestimated. In the model by Chiappini et al. (1997) (our Model 1) $[\mathrm{Fe} / \mathrm{H}]$ reaches a local peak at $1 \mathrm{Gyr}$, then decreases slightly to increase again. The small depression in $[\mathrm{Fe} / \mathrm{H}]$ is due to the predicted gap in the SFR just before the formation of the thin disk. The second infall episode coupled with the halt in the SF produces a decrease of $[\mathrm{Fe} / \mathrm{H}]$. We can see the same behaviour in the cosmological models. In particular in Model 5 the peak is followed by a deeper depression of $[\mathrm{Fe} / \mathrm{H}]$ and this is due to the longer gap in the SFR predicted by this model (1-2 Gyr) as opposed to that predicted by Model 1, which is less than 1 Gyr. This is an important prediction and it can be tested via chemical abundances. Both Gratton et al. (1996) and Furhmann (1998) detected such an effect in the $[\mathrm{Fe} / \mathrm{O}]$ vs. $[\mathrm{O} / \mathrm{H}]$ and $[\mathrm{Fe} / \mathrm{Mg}]$ vs. $[\mathrm{Mg} / \mathrm{H}]$, respectively. 


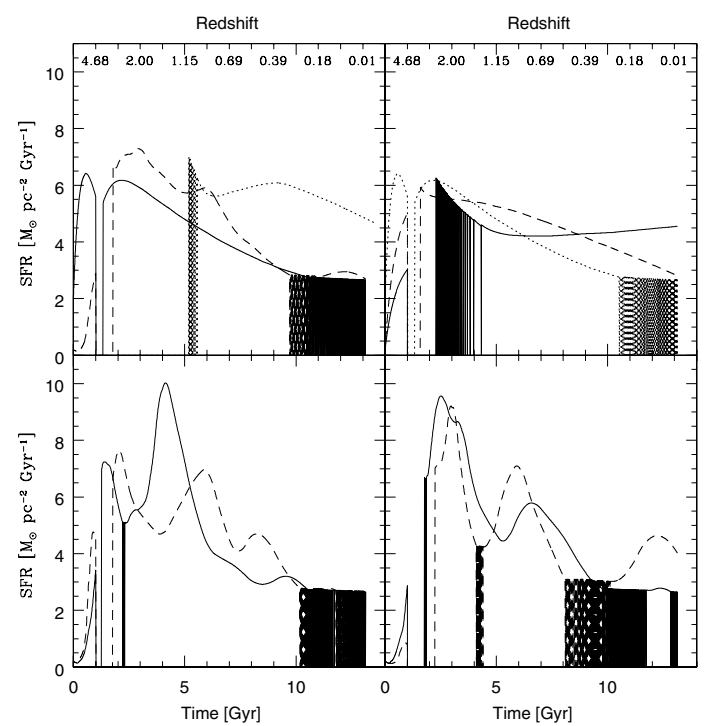

Fig. 6. SFR vs. time. Upper left panel: red solid line is the two-infall model (Model 1); black dashed line is the cosmological mean model (Model 9); green dotted line is the model by Naab \& Ostriker (2006) (Model 10). Upper right panel: magenta solid line is the constant infall model (Model 2); blue dashed line is the linear infall model (Model 3); cyan dotted line is the pre-enriched model $\left(Z_{\text {inf }}=1 / 10 Z_{\text {today }}\right.$, Model 4$)$. Bottom left panel: black solid line is Model 5; magenta dashed line is Model 6. Bottom right panel: blue solid line is Model 7; cyan dashed line is Model 8.

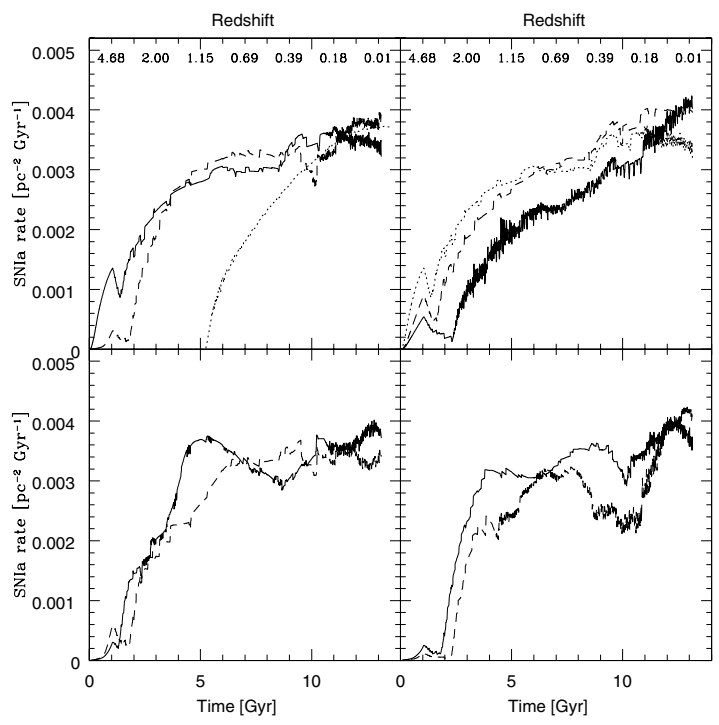

Fig. 7. SNIa rate vs. time. Upper left panel: red solid line is the twoinfall model (Model 1); black dashed line is the cosmological mean model (Model 9); green dotted line is the model by Naab \& Ostriker (2006) (Model 10). Upper right panel: magenta solid line is the constant infall model (Model 2); blue dashed line is the linear infall model (Model 3); cyan dotted line is the pre-enriched model $\left(Z_{\text {inf }}=\right.$ $1 / 10 Z_{\text {today }}$, Model 4). Bottom left panel: black solid line is Model 5; magenta dashed line is Model 6. Bottom right panel: blue solid line is Model 7; cyan dashed line is Model 8.

A very important constraint for the chemical evolution of the galaxies is represented by the G-dwarf metallicity distribution. This is the relative number of G-dwarf stars as a function of $[\mathrm{Fe} / \mathrm{H}]$. We have used the data from Rocha-Pinto \& Maciel (1996), Kotoneva (2002), Jorgensen (2000) and Wyse (1995). Our predicted metallicity distributions are shown in Fig. 9. From

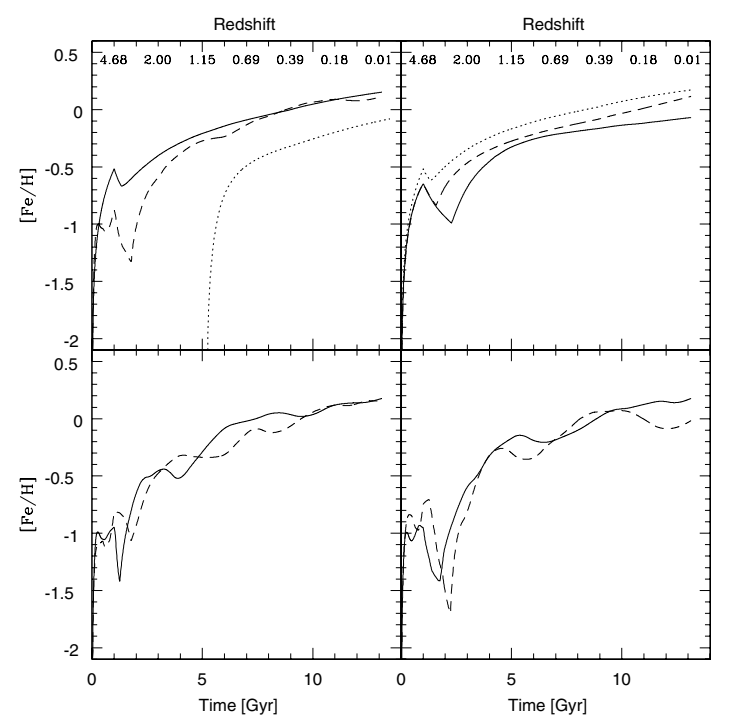

Fig. 8. $[\mathrm{Fe} / \mathrm{H}]$ vs. time. Upper left panel: red solid line is the two-infall model (Model 1); black dashed line is the cosmological mean model (Model 9); green dotted line is the model by Naab \& Ostriker (2006) (Model 10). Upper right panel: magenta solid line is the constant infall model (Model 2); blue dashed line is the linear infall model (Model 3); cyan dotted line is the pre-enriched model $\left(Z_{\text {inf }}=1 / 10 Z_{\text {today }}\right.$, Model 4$)$. Bottom left panel: black solid line is Model 5; magenta dashed line is Model 6. Bottom right panel: blue solid line is Model 7; cyan dashed line is Model 8.

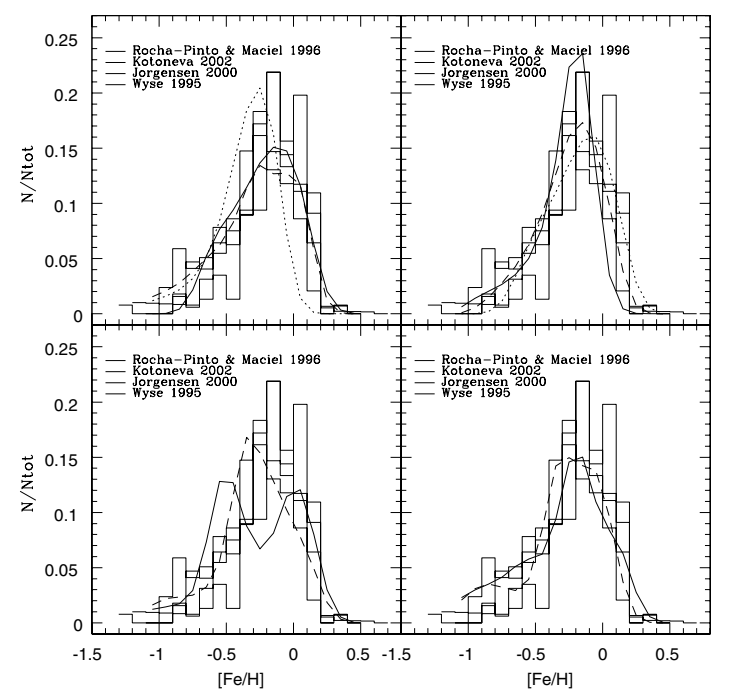

Fig. 9. G-dwarf metallicity distribution. Upper left panel: red solid line is the two-infall model (Model 1); black dashed line is the cosmological mean model (Model 9); green dotted line is the model by Naab \& Ostriker (2006) (Model 10). Upper right panel: magenta solid line is the constant infall model (Model 2); blue dashed line is the linear infall model (Model 3); cyan dotted line is the pre-enriched model $\left(Z_{\text {inf }}=1 / 10 Z_{\text {today }}\right.$, Model 4). Bottom left panel: black solid line is Model 5; magenta dashed line is Model 6. Bottom right panel: blue solid line is Model 7; cyan dashed line is Model 8.

this figure, it is clear that Model 10 predicts insufficient high metallicity stars. On the other hand, some of the cosmological models such as Model 7 and Model 8 predict too many metalpoor stars. Our best cosmological model, i.e. Model 5, shows a bimodal metallicity distribution, which is clearly at odds with the data. 


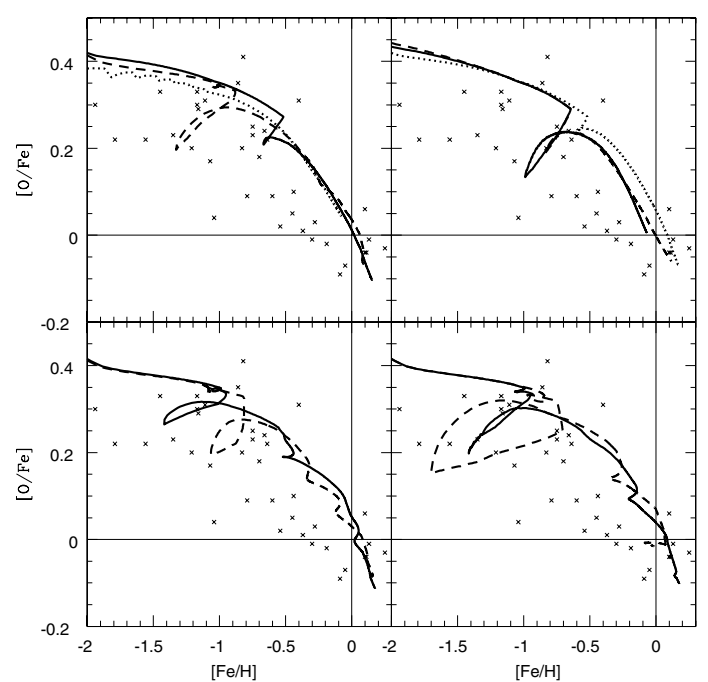

Fig. 10. $[\mathrm{O} / \mathrm{Fe}]$ vs. $[\mathrm{Fe} / \mathrm{H}]$. Upper left panel: red solid line is the twoinfall model (Model 1); black dashed line is the cosmological mean model (Model 9); green dotted line is the model by Naab \& Ostriker (2006) (Model 10). Upper right panel: magenta solid line is the constant infall model (Model 2); blue dashed line is the linear infall model (Model 3); cyan dotted line is the pre-enriched model $\left(Z_{\text {inf }}=\right.$ $1 / 10 Z_{\text {today }}$, Model 4). Bottom left panel: black solid line is Model 5; magenta dashed line is Model 6. Bottom right panel: blue solid line is Model 7; cyan dashed line is Model 8. The data are from François et al. (2004) (green crosses).

The last constraint we study concerns the chemical abundances of several elements, such as $\mathrm{O}, \mathrm{Mg}, \mathrm{Si}, \mathrm{N}$ and $\mathrm{C}$. In Fig. 10 the $[\mathrm{O} / \mathrm{Fe}]$ as a function of $[\mathrm{Fe} / \mathrm{H}]$ can be seen. Here, the range of $[\mathrm{Fe} / \mathrm{H}]$ has been restricted to -2.0 to +0.3 dex in order to better see the predictions relative to the transition between the halo-thick disk and the thin disk. In Fig. 11 we show the same plots but for the whole range of $[\mathrm{Fe} / \mathrm{H}]$ down to -4.0 dex.

In Fig. 10 one can see that cosmological models have a similar behaviour to the model by Chiappini et al. (1997), except for a longer gap in the SF, which produces a loop in the predicted curves. Such loops arise when SF stops, the $\alpha$-elements are no longer produced whereas Fe continues to be produced. This induces the $[\mathrm{O} / \mathrm{Fe}]$ to decrease and also the $[\mathrm{Fe} / \mathrm{H}]$ ratio to decrease to a lesser extent, because of the accretion of primordial gas. Then when SF starts again the $[\mathrm{O} / \mathrm{Fe}]$ again increases. This loop is very prominant in some models and is not in agreement with the data, although some spread is present. Model 4, which is the same as Chiappini et al.'s model but with the pre-enriched gas, is acceptable. This is due to the fact that the metallicity of the pre-enriched infalling gas is not so different from the metallicity of the primordial infalling gas.

Figures 12 and 13 present the $[\mathrm{Mg} / \mathrm{Fe}]$ and the $[\mathrm{Si} / \mathrm{Fe}]$ as a function of $[\mathrm{Fe} / \mathrm{H}]$. The data in Figs. 10-13 are from Cayrel et al. (2004) for the very metal poor stars and from the compilation of François et al. (2004) for all the others. Once again all the considerations made above for $[\mathrm{O} / \mathrm{Fe}]$ are valid for these other $\alpha$-elements.

Two other important elements are $\mathrm{C}$ and N. Figures 14 and 15 show the behaviour of $[\mathrm{C} / \mathrm{Fe}]$ and $[\mathrm{N} / \mathrm{Fe}]$ as a function of $[\mathrm{Fe} / \mathrm{H}]$. The data in Fig. 14 are from Spite et al. (2005) (magenta points), Carbon et al. (1987) (red points), Clegg et al. (1981) (cyan points), Laird (1985a,b) (black points) and Tomkin et al. (1995) (green points). Figure 15 presents the data from Spite et al. (2005) (magenta points), Israelian et al. (2004) (blue points), Carbon et al. (1987) (red points), Clegg et al. (1981)

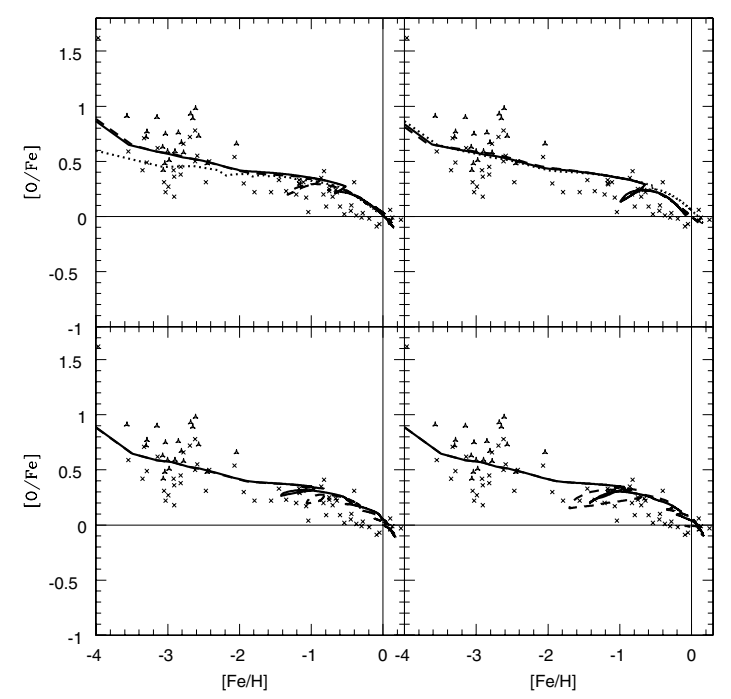

Fig. 11. $[\mathrm{O} / \mathrm{Fe}]$ vs. $[\mathrm{Fe} / \mathrm{H}]$. Upper left panel: red solid line is the twoinfall model (Model 1); black dashed line is the cosmological mean model (Model 9); green dotted line is the model by Naab \& Ostriker (2006) (Model 10). Upper right panel: magenta solid line is the constant infall model (Model 2); blue dashed line is the linear infall model (Model 3); cyan dotted line is the pre-enriched model $\left(Z_{\text {inf }}=\right.$ $1 / 10 Z_{\text {today }}$, Model 4). Bottom left panel: black solid line is Model 5; magenta dashed line is Model 6. Bottom right panel: blue solid line is Model 7; cyan dashed line is Model 8. The data are from: Cayrel et al. (2004) (red triangles) and François et al. (2004) (green crosses).

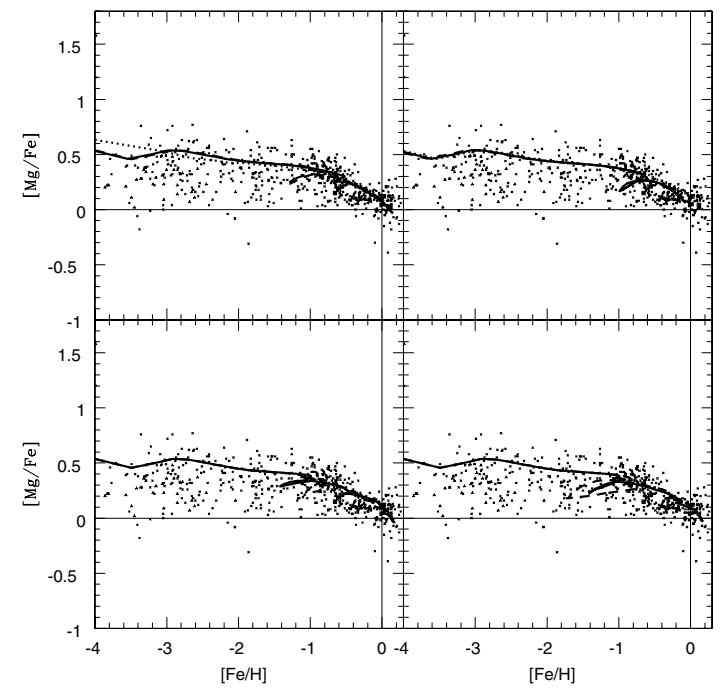

Fig. 12. $[\mathrm{Mg} / \mathrm{Fe}]$ vs. $[\mathrm{Fe} / \mathrm{H}]$. Upper left panel: red solid line is the two-infall model (Model 1); black dashed line is the cosmological mean model (Model 9); green dotted line is the model by Naab \& Ostriker (2006) (Model 10). Upper right panel: magenta solid line is the constant infall model (Model 2); blue dashed line is the linear infall model (Model 3); cyan dotted line is the pre-enriched model $\left(Z_{\text {inf }}=1 / 10 Z_{\text {today }}\right.$, Model 4$)$. Bottom left panel: black solid line is Model 5; magenta dashed line is Model 6. Bottom right panel: blue solid line is Model 7; cyan dashed line is Model 8. The data are from: Cayrel et al. (2004) (red triangles) and François et al. (2004).

(cyan points) and Laird (1985a,b) (black points). From Fig. 14 it can be seen once again that the cosmological models are very similar to the model by Chiappini et al. (1997). The predicted curves are different only for values of $[\mathrm{Fe} / \mathrm{H}]$ higher than -1.5 dex. The same holds for the $[\mathrm{N} / \mathrm{Fe}]$. In both cases, cosmological models have a particular behaviour at high metallicities. 


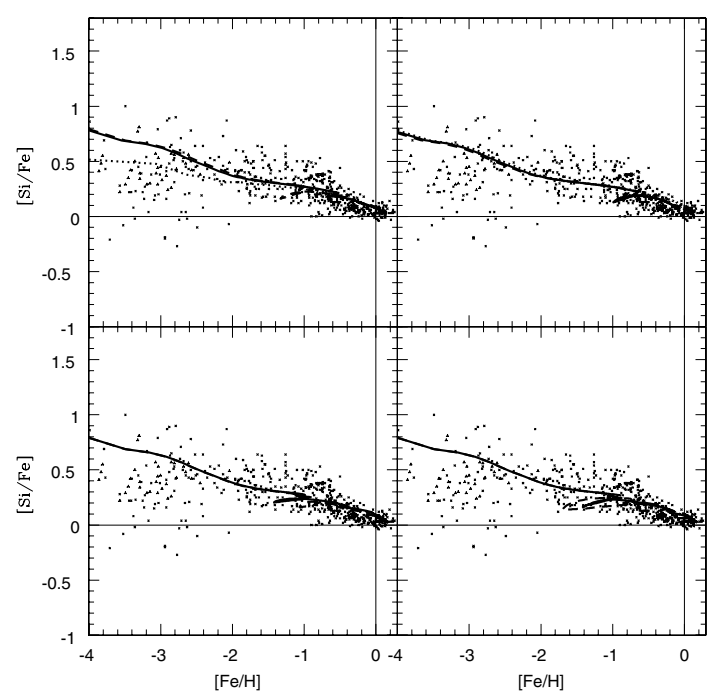

Fig. 13. $[\mathrm{Si} / \mathrm{Fe}]$ vs. $[\mathrm{Fe} / \mathrm{H}]$. Upper left panel: red solid line is the twoinfall model (Model 1); black dashed line is the cosmological mean model (Model 9); green dotted line is the model by Naab \& Ostriker (2006) (Model 10). Upper right panel: magenta solid line is the constant infall model (Model 2); blue dashed line is the linear infall model (Model 3); cyan dotted line is the pre-enriched model $\left(Z_{\text {inf }}=\right.$ 1/10 $Z_{\text {today }}$, Model 4). Bottom left panel: black solid line is Model 5; magenta dashed line is Model 6. Bottom right panel: blue solid line is Model 7; cyan dashed line is Model 8. The data are from: Cayrel et al. (2004) (red triangles) and François et al. (2004).

This behaviour is common to all the elements analysed and is due to the gap in the SFR at about 1 Gyr, as discussed before. In the cosmological models this effect is larger because of the longer duration of the gap. However in the case of $[\mathrm{C} / \mathrm{Fe}]$ and $[\mathrm{N} / \mathrm{Fe}]$ we cannot draw any firm conclusion because of the large spread in the data. In Fig. 16 we show the $\mathrm{O}$ abundance gradient as predicted by Model 1 and Model 5, compared with a compilation of data including Cepheids (see Cescutti et al. 2007). As one can see, the $\mathrm{O}$ gradient predicted by Model 5 flattens for $r<8 \mathrm{kpc}$ whereas it agrees very well with the slope predicted by Model 1 (the original two-infall model) for $r \geq 8 \mathrm{kpc}$. Model 1 contains the assumption of an inside-out formation of the disk, as described by Eq. (3), whereas in Model 5 no such assumption is made. In spite of that, the two predicted gradients are similar and we cannot reject the $\mathrm{O}$ gradient predicted by Model 5 on the basis of the comparison with data. The reason for that probably resides in the adoption of the star formation threshold which acts mainly at large galactocentric distances where the gas density is lower. This effect predominates over the increase of the timescale for disk formation. This deserves more detailed study which we plan in more detail of the disk evolution in a cosmological context.

Figures 17-19 present the results obtained by using a different infall law, derived from the cosmological simulation but selecting different parameters. In this case we selected a halo which is not expected to produce a spiral galaxy, so we looked for a spin parameter lower than 0.04 , a redshift of the last major merger lower than 2.5 and a redshift of formation lower than 1.0. Such a halo is perhaps more appropriate for an elliptical or So galaxy. We found a halo with the following characteristics:

- mass $=2.15 \times 10^{12} M_{\odot}$;

$-\lambda=0.029$;

- redshift of major merger $=0.50$;

- redshift of formation $=0.75-0.63$.

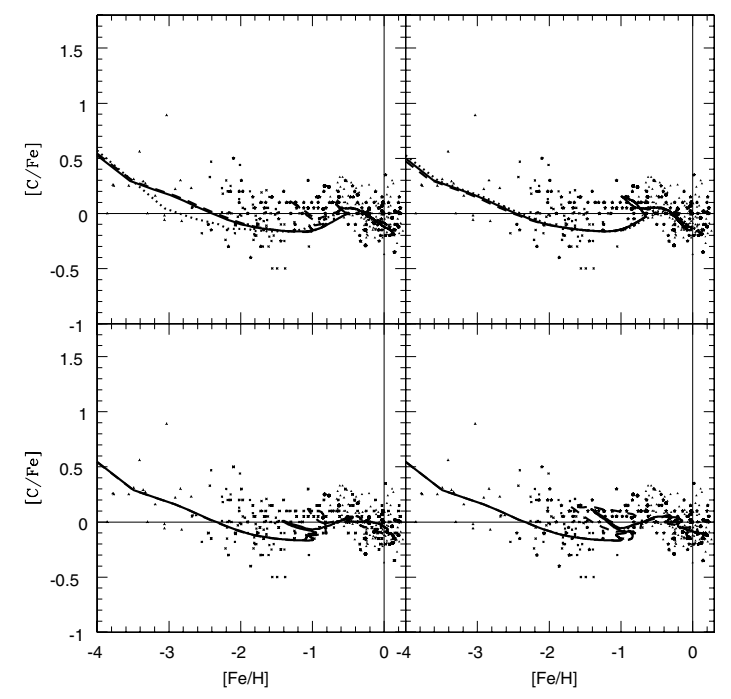

Fig. 14. $[\mathrm{C} / \mathrm{Fe}]$ vs. $[\mathrm{Fe} / \mathrm{H}]$. Upper left panel: red solid line is the twoinfall model (Model 1); black dashed line is the cosmological mean model (Model 9); green dotted line is the model by Naab \& Ostriker (2006) (Model 10). Upper right panel: magenta solid line is the constant infall model (Model 2); blue dashed line is the linear infall model (Model 3); cyan dotted line is the pre-enriched model $\left(Z_{\text {inf }}=\right.$ $1 / 10 Z_{\text {today }}$, Model 4). Bottom left panel: black solid line is Model 5; magenta dashed line is Model 6. Bottom right panel: blue solid line is Model 7; cyan dashed line is Model 8. The data are from: Spite et al. (2005) (magenta stars with three arms), Carbon et al. (1987) (red crosses), Clegg et al. (1981) (cyan circles), Laird (1985a,b) (black stars with five arms) and Tomkin et al. (1995) (green triangles).

Figures 17-19 compare the results from this halo with Model 1 (two-infall law) and Model 5 (our best cosmological choice). The infall law is very different. In particular, it has a major peak at a redshift of about 0.3 . This produces a peak at the same redshift in the star formation rate and, of course, in the SNII rate. Moreover, there is a strong depression in the $[\mathrm{Fe} / \mathrm{H}]$ ratio between 1.8 and 3 Gyr from the beginning of the simulation, difficult to reconcile with observations.

In Figs. 18 and 19 we show the results for the $[\mathrm{O} / \mathrm{Fe}]$ and for the G-dwarf metallicity distribution. The main difference between this halo and Models 1 and 5 is that the loop placed at $[\mathrm{Fe} / \mathrm{H}] \sim-1.0$ is longer and predicts low values of $[\mathrm{O} / \mathrm{Fe}]$ at low $[\mathrm{Fe} / \mathrm{H}]$, which is not observed in Galactic stars. As far as the G-dwarf metallicity distribution is concerned, the halo forms too many stars with low metallicity as a consequence of the deep depression in the $[\mathrm{Fe} / \mathrm{H}]$ ratio (see the plot on the bottom right part of Fig. 17), again not in agreement with the data, and resembles an early-type galaxy. This example confirms the importance of the cosmological assembly history of the DM halo in determining not only the morphological parameters of the galaxy it hosts, but also its chemical properties.

\section{Conclusions}

We have tested different gas infall laws for models of the formation of the Milky Way and especially cosmologically derived infall laws, obtained by means of cosmological simulations for the formation of the DM halo of the Milky Way. In particular, we assumed that the accretion law for the DM halo holds also for the baryonic matter. We found four different DM halos with properties compatible with a disk galaxy, with one in particular seemed better than the others. All these infall laws were then compared with the one proposed by Chiappini et al. (1997), called the 


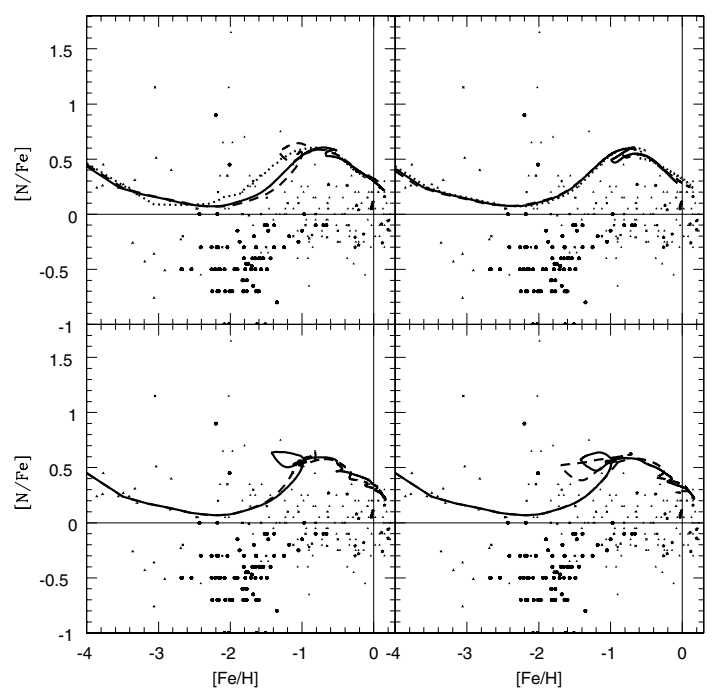

Fig. 15. $[\mathrm{N} / \mathrm{Fe}]$ vs. $[\mathrm{Fe} / \mathrm{H}]$. Upper left panel: red solid line is the twoinfall model (Model 1); black dashed line is the cosmological mean model (Model 9); green dotted line is the model by Naab \& Ostriker (2006) (Model 10). Upper right panel: magenta solid line is the constant infall model (Model 2); blue dashed line is the linear infall model (Model 3); cyan dotted line is the pre-enriched model $\left(Z_{\text {inf }}=\right.$ $1 / 10 Z_{\text {today }}$, Model 4). Bottom left panel: black solid line is Model 5; magenta dashed line is Model 6. Bottom right panel: blue solid line is Model 7; cyan dashed line is Model 8. The data are from: Spite et al. (2005) (magenta stars with three arms), Israelian et al. (2004) (blue crosses), Carbon et al. (1987) (red circles), Clegg et al. (1981) (cyan stars with five arms) and Laird (1985a,b) (black triangles).

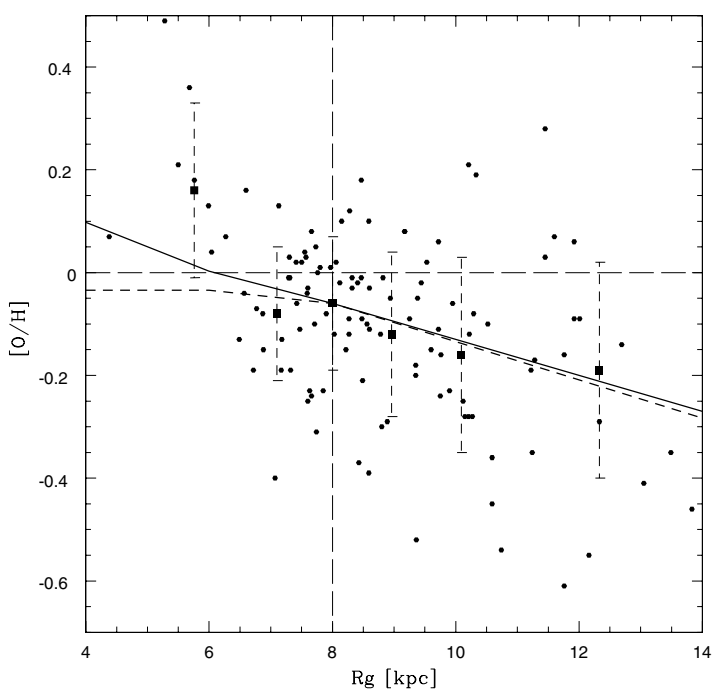

Fig. 16. Predicted and observed $\mathrm{O}$ abundance gradients in the galactocentric distance range $4-14 \mathrm{kpc}$. The continuous line is the prediction of the two-infall model, the dashed line is the prediction of Model 5. The data points are from Cepheids. The large squares with error bars represent averages of the points with their errors (see Cescutti et al. 2007, and reference therein).

two-infall law, which predicts that there were two main accretion episodes which formed the halo-bulge-thick disk and the thin disk, respectively. We found that our best cosmological infall law is very similar to the two-infall one, which has already proven to be able to reproduce the majority of the chemical properties of the Milky Way in the solar neighbourhood. Our cosmological infall laws have been tested in a detailed chemical evolution model for the Milky Way, following the evolution of several chemical
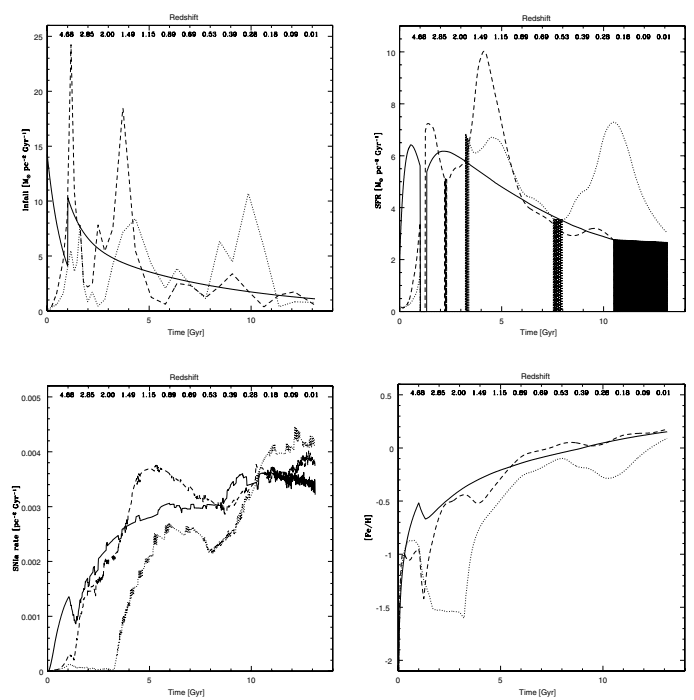

Fig. 17. The infall law (upper left panel), the star formation rate (upper right panel), the SNIa rate (bottom left panel) and the $[\mathrm{Fe} / \mathrm{H}]($ bottom right panel) as a function of time for the two-infall model (Model 1, red solid line), for Model 5 (black dashed line) and for the halo 20912 (blue dotted line).

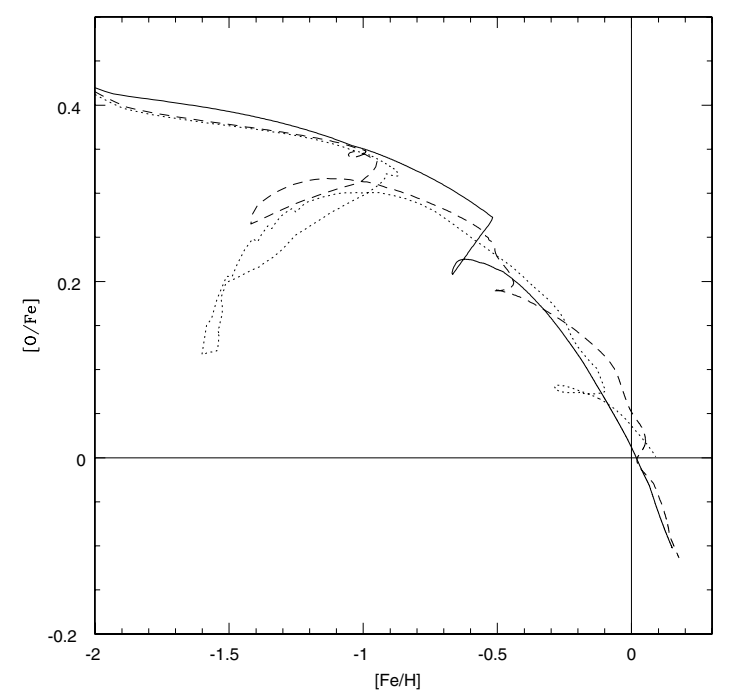

Fig. 18. This plot represents the $[\mathrm{O} / \mathrm{Fe}]$ as a function of $[\mathrm{Fe} / \mathrm{H}]$. The red solid line represents the two-infall model (Model 1), the black dashed line represents Model 5 and the blue dashed line the halo 20912.

elements by taking into account stellar lifetimes, SN progenitors and stellar nucleosynthesis.

Our main conclusions can be summarized as follows:

- A model with constant infall predicts a present day infall rate and SFR larger than all the other models. Moreover, it is the only model that produced an unrealistically increasing SFR during the last billion years. This is probably an unrealistic law, and we only used it for comparison with other infall laws.

- The linear model predicts the largest number of stars presently in the solar neighbourhood but it seems to reproduce reasonably well all the other observables. However, this model does not describe the evolution of our Galaxy as well as an exponential law does.

- The model adopting the two-infall law, but where the gas is assumed to be pre-enriched during the formation of the disk 


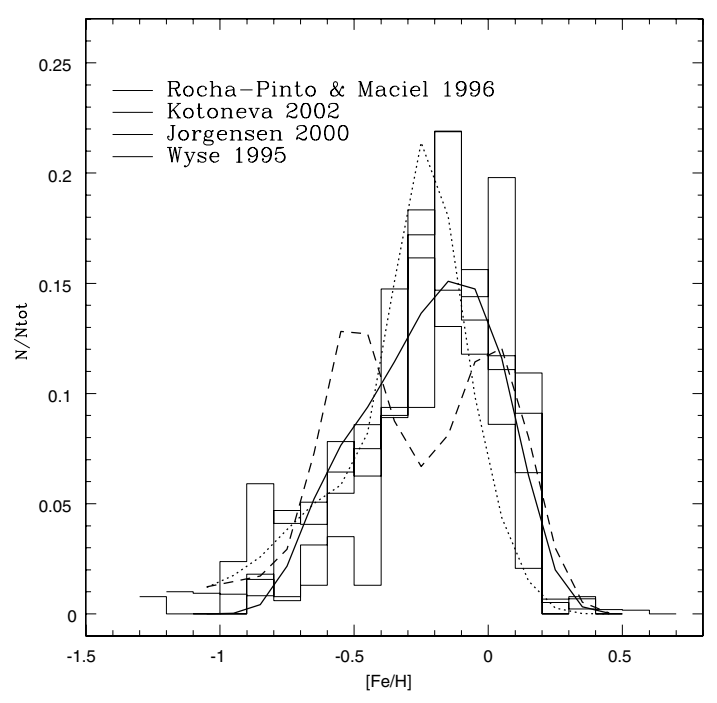

Fig. 19. G-dwarf metallicity distribution for the two-infall model (red solid line), Model 5 (black dashed line) and for the halo 20912 (blue dashed line).

at the level of $1 / 10$ of solar, well reproduces the G-dwarf metallicity distribution, as expected.

- The cosmological laws, and in particular our preferred best fit, seem to fit well all the data. This law predicts two main accretion episodes which can be identified with the formation of the halo-thick disk and thin disk, respectively, very similar to the two-infall law. Moreover, there seems to be a gap of 1-2 Gyr in the SFR between the two episodes, larger than predicted by Chiappini et al. (1997) $(<1$ Gyr). The gap is due mainly to the adoption of a threshold gas density for the star formation rate. Such a gap seems to have been observed looking at abundance patterns, in particular at $[\mathrm{Fe} / \mathrm{O}]$ vs. $[\mathrm{O} / \mathrm{H}]$ (Gratton et al. 1996) and at [Fe/Mg] vs. [Fe/H] (Fuhrmann 1998), although new data are necessary to draw firm conclusions. The model including this cosmological infall law can well reproduce most of the observational constraints. It predicts for the G-dwarf metallicity distribution, in the solar vicinity, two different peaks: we speculate that the first peak represents the stars of the halo and thick disk while the second peak represents the stars of the thin disk. The same metallicity distribution computed for the central region should also include the bulge stars. The predicted timescales for the formation of the halo-thick disk and the thin disk, respectively, are in excellent agreement with those suggested by Chiappini et al. In particular, the halothick disk must have formed on a timescale not longer than 1-2 Gyr whereas the thin disk in the solar vicinity took at least 6 Gyr to assemble $60 \%$ of its mass. As a consequence of the gap between the halo-thick disk and the thin disk, we predict that the thin disk is at least 2 Gyr younger than the halo.

- The other cosmological infall laws are characterized by several minor accretion events after the two main ones and predict larger gaps in the SFR which are not observed in the $[\mathrm{Fe} / \mathrm{O}]$ vs. $[\mathrm{O} / \mathrm{H}]$ and $[\mathrm{Fe} / \mathrm{Mg}]$ vs. $[\mathrm{Mg} / \mathrm{H}]$ which indicate a gap not larger than 1-2 Gyr.

- A model adopting a cosmologically inferred infall law by Naab \& Ostriker (2006) presents a behaviour very similar to the constant infall law and predicts too low metallicities at the Sun's age and at the present time. Moreover, this model predicts a too small number of G-dwarfs with high metallicity. In their paper they present a G-dwarf metallicity distribution but as a function of $\mathrm{Z}$ which represents $\mathrm{O}$ and not $\mathrm{Fe}$, as in the observations.

- Our results strongly depend on what criteria were used to select the dark matter halo from the cosmological simulations. If they are not suitable for forming a spiral galaxy it is possible to see that the results are not in good agreement with the observations. We prove this by using a DM halo with dynamical parameters compatible with an early-type galaxy.

- Our results can be compared with the work of Robertson et al. (2005), in which the authors studied the chemical enrichment of the stellar halo of the Milky Way, using the prescriptions of the hierarchical scenario. They supposed that most of the mass in the MW halo was acquired via mergers with massive dIrr-type DM halos, occurring at a look-back time of 10 Gyr. They used three examples of mass accretion history, supposing that the cumulative mass accretion in individual DM halos can be well described by an analytical function obtained by Wechsler et al. (2002).

Moreover, they assumed that the cold gas inflow rate tracks the DM accretion rate and that the fraction of cold gas is equal to $2 \%$.

In order to build the stellar halo of the Milky Way they used a dIrr-type dark matter halo with a virial mass $M_{0}=6 \times$ $10^{10} M_{\odot}$, accreted $9 \mathrm{Gyr}$ ago, following their assumed accretion law. In this case the time available for the star formation and the consequent chemical enrichment is only $\sim 2.6 \mathrm{Gyr}$ and therefore the chemical enrichment due to SNIa was limited. We do not use the accretion of a dIrr galaxy to build the stellar halo of the MW. We obtained the mass accretion history of the DM halo directly from the cosmological simulation, done with the public tree-code GADGET2 (Springel 2005). For this reason we accrete only DM and cold gas and not already formed dwarf galaxies, with their own stars and gas. Moreover we study the chemical enrichment of all the galaxy and not only of the stellar halo.

- In the future we plan to extend the current work, and in particular our cosmologically derived baryonic infall laws, to the study of the chemical properties of the whole disk. As we have already shown in this paper, by normalizing the infall law to the current total surface mass density along the disk, we obtain different timescales for the assembly of the disk as a function of galactocentric distance, although the inside-out effect is not as marked as in the Matteucci \& François (1989) and Chiappini et al. (2001) models.

The fact that all our four suitable DM halos show an accretion law that resembles that used in the two-infall model could be linked to the way in which such halos assemble. Indeed, they have their last major merger at high redshift, larger than $z=2.5$, by selection and they reach a mass larger than $50 \%$ of their final one at lower redshift. As a consequence of our requirement not to have late major mergers, such late assembly happens via accretion of material from the field, namely filaments, or via minor mergers. These two epochs of important accretion qualitatively correspond to the two peaks used in the two-infall model and give it a cosmological motivation. Obviously the details of the late accretion episode will depend on the dynamical history of the single DM halo, and will generate differences in the chemical patterns of individual late-type galaxies without destroying their overall properties. On the other hand, halos should acquire their angular momentum thanks to the cosmological torques acting at high redshifts on the material (both baryons and dark matter) which will coalesce to form them. Such torques will also influence their mass accretion histories. Thus, selecting DM halos 
with high spin values could also result in selecting halos with similar dynamical histories. Astrophysical processes acting on baryons, e.g. feedback, should not be able to dramatically alter this scenario. Finally we note that, while in the two-infall model the timing of the two episodes is a free parameter, in the cosmological infall scenario the timing is directly given by the gravitational evolution of the halos. In this sense, the agreement between such models is not a-priori guaranteed and could be interpreted as an interesting link between the morphological properties of the late-type galaxies (used to fix our requirement) and their chemical properties, via the hierarchical model.

Acknowledgements. We thank Gabriele Cescutti and Cristina Chiappini for some helpful suggestions and Donatella Romano, Patrick François and Silvia Kuna Ballero for their collaboration concerning the data. We also thank the referee Chris Flynn for valuable comments.

\section{References}

Abadi, M. G., Navarro, J. F., Steinmetz, M., \& Eke, V. R. 2003a, ApJ, 591, 499 Abadi, M. G., Navarro, J. F., Steinmetz, M., \& Eke, V. R. 2003b, ApJ, 597, 21 Andrievsky, S. M., Luck, R. E., Martin, P., \& Lépine, J. R. D. 2004, A\&A, 413, 159

Asplund, M., Grevesse, N., \& Sauval, A. J. 2005, ASPC, 336, 25

Beers, T. C., \& Sommer-Larsen, J. 1995, ApJS, 96, 175

Bensby, T., Feltzing, S., \& Lundström, I. 2003, A\&A, 410, 527

Bertschinger, E. 1995 [arXiv:astro-ph/6070]

Boissier, S., \& Prantzos, N. 1999, MNRAS, 307, 857

Boissier, S., \& Prantzos, N. 2000, MNRAS, 312, 398

Boissier, S., Boselli, A., Prantzos, N., \& Gavazzi, G. 2001, MNRAS, 321, 733

Boissier, S., Gil de Paz, A., Boselli, A., et al. 2006 [arXiv: astro-ph/0609071]

Bryan, G. L., \& Norman, M. L. 1997, ASPC, 123, 363

Bullock, J. S., Kolat, T. S., Sigad, Y., et al. 2001, MNRAS, 321, 559

Burkert, A., Truran, J. W., \& Hensler, G. 1992, ApJ, 391, 651

Carbon, D. F., Barbuy, B., Kraft, R. P., Friel, E. D., \& Suntzeff, N. B. 1987, PASP, 99, 335

Carigi, L. 1994, ApJ, 424, 181

Carney, B. W., Wright, J. S., Sneden, C., et al. 1997, AJ, 114, 363

Carretta, E., Gratton, R., Cohen, J. G., Beers, T. C., \& Christlieb, N. 2002, AJ, 124,481

Cayrel, R., Depagne, E., Spite, M., et al. 2004, A\&A, 416, 1117

Cescutti, G., François, P., Matteucci, F., Cayrel, R., \& Spite, M. 2006, A\&A, 448,557

Cescutti, G., Matteucci, F., François, P., \& Chiappini, C. 2007, A\&A, 462, 943

Chiappini, C., Matteucci, F., \& Gratton, R. 1997, ApJ, 477, 765

Chiappini, C., Matteucci, F., Beers, T. C., \& Nomoto, K. 1999, ApJ, 515, 226

Chiappini, C., Matteucci, F., \& Romano, D. 2001, ApJ, 554, 1044

Chiosi, C. 1980, A\&A, 83, 206

Clegg, R. E. S., Lambert, D. L., \& Tomkin, J. 1981, ApJ, 250, 262

Cowie, L. L., Songaila, A., \& Hu, E. M. 1996, AJ, 112, 839

Davis, M., Efstathiou, G., Frenk, G. S., \& White, S. D. M. 1985, ApJ, 292, 371

D’Onghia, E., \& Burkert, A. 2004, ApJ, 612, 13

D’Onghia, E., Burkert, A., Murante, G., \& Khochfar, S. 2006, MNRAS, 372, 1525

Dopita, M. A., \& Ryder, S. D. 1994, ApJ, 430, 163

Efstathiou, G., Davies, M., White, S. D. M., \& Frenk, G. S. 1985, ApJS, 57, 241

Ferrini, F., Molla, M., Pardi, M. C., \& Diaz, A. I. 1994, ApJ, 427, 745

François, P., Matteucci, F., Cayrel, R., et al. 2004, A\&A, 421, 613

Fulbright, J. P. 2000, AJ, 120, 1841

Fuhrnamm, K. 1998, A\&A, 338, 161

Gilmore, G., Wyse, R. F. G., \& Jones, J. B. 1995, AJ, 109, 1095

Gilroy, K. K., Sneden, C., Pilachowski, C. A., \& Cowan, J. J. 1988, ApJ, 327, 298

Giovagnoli, A., \& Tosi, M. 1995, MNRAS, 273, 499

Governato, F., Mayer, L., Wadsley, J., et al. 2004, ApJ, 607, 688

Governato, F., Willman, B., Mayer, L., et al. 2007, MNRAS, 374, 1479

Gratton, R., \& Sneden, C. 1988, A\&A, 204, 193

Gratton, R., \& Sneden, C. 1994, A\&A, 287, 927

Gratton, R., Carretta, E., Matteucci, F., \& Sneden, C. 1996, ASPC, 92, 371

Guzmán, R., Gallego, J., Koo, D. C., et al. 1997, ApJ, 489, 559

Han, Z., \& Podsiadlowski, P. 2004, MNRAS, 350, 1301

Hou, J. L., Prantzos, N., \& Boissier, S. 2000, A\&A, 362, 921
Israelian, G., Ecuvillo, A., Rebolo, R., et al. 2004, A\&A, 421, 649

Iwamoto, K., Brachwitz, F., Nomoto, K., et al. 1999, ApJS, 125, 439

Jørgensen, B. R. 2000, A\&A, 363, 947

Kauffmann, G. 2001, ASPC, 245, 381

Kennicutt, R. C. Jr. 1998, ARA\&A, 36, 189

Klypin, A., \& Primack, J. R. 2001, ApJ, 554, 903

Kotoneva, E., Flynn, C., Chiappini, C., \& Matteucci, F. 2002, MNRAS, 336, 879

Lacey, C. G., \& Fall, S. M. 1985, ApJ, 290, 154

Laird, J. B. 1985a, ApJS, 57, 389

Laird, J. B. 1985b, ApJS, 289, 556

Lanfranchi, G., \& Matteucci, F. 2004, MNRAS, 351, 1338

Larson, R. B. 1976, MNRAS, 176, 31

Malinie, G., Hartmann, D. H., Clayton, D. D., \& Mathews, G. J. 1993, ApJ, 413, 633

Matteucci, F. 1994, A\&A, 288, 57

Matteucci, F., \& Greggio, L. 1986, A\&A, 154, 279

Matteucci, F., \& François, P. 1989, MNRAS, 239, 885

Matteucci, F., \& François, P. 1992, A\&A, 262, 1

Matteucci, F., Raiteri, C. M., Busson, M., Gallino, R., \& Gratton, R. 1993, A\&A, 272,421

McWilliam, A., Preston, G. W., Sneden, C., \& Searle, L. 1995, AJ, 109, 2757

Naab, T., \& Ostriker, J. P. 2006, MNRAS, 366, 899

Nagashima, M., \& Okamoto, T. 2006, ApJ, 643, 863

Navarro, J. F., \& Steinmetz, M. 2000, ApJ, 538, 477

Navarro, J. F., Frenk, C. S., \& White, S. D. M. 1995, MNRAS, 275, 56

Navarro, J. F., Frenk, C. S., \& White, S. D. M. 1996, ApJ, 462, 563

Navarro, J. F., Frenk, C. S., \& White, S. D. M. 1997, ApJ, 490, 493

Nissen, P. E., \& Schuster, W. J. 1997, A\&A, 326, 751

Nissen, P. E., Primas, F., \& Asplund, D. L. M. L. 2002, A\&A, 390, 235

Pagel, B. E. J. 1989, in Evolutionary phenomena in galaxies (Cambridge University Press), 201

Pardi, M. C., \& Ferrini, F. 1994, ApJ, 421, 491

Pardi, M. C., Ferrini, F., \& Matteucci, F. 1995, ApJ, 444, 207

Pipino, A., \& Matteucci, F. 2004, MNRAS, 347, 968

Power, C., Navarro, J. F., Jenkins, A., et al. 2003, MNRAS, 338, 14

Prantzos, N., \& Aubert, O. 1995, A\&A, 302, 69

Prochaska, J. X., Naumov, S. O., Carney, B. W., McWilliam, A., \& Wolfe, A. M. 2000, AJ, 120, 2513

Raiteri, C. M., Villata, M., \& Navarro, J. F. 1996a, A\&A, 315, 105

Raiteri, C. M., Villata, M., \& Navarro, J. F. 1996b, MmSaIt, 67, 817

Raiteri, C. M., Villata, M., Gallino, R., Busso, M., \& Cravanzola, A. 1999, ApJ, 518, 91

Recchi, S., Matteucci, F., D’Ercole, A., \& Tosi, M. 2002, A\&A, 384, 799

Reid, M. J. 1993, ARA\&A, 31, 345

Robertson, B., Yoshida, N., Springel, V., \& Hernquist, L. 2004, ApJ, 606, 32

Robertson, B., Bullock, J. S., Font, A. S., Johnston, K. V., \& Hernquist, L. 2005 , ApJ, 632, 872

Rocha-Pinto, H. J., \& Maciel, W. J. 1996, MNRAS, 279, 447

Ryan, S., Norris, J. E., \& Bessell, M. S. 1991, AJ, 102, 303

Samland, M., \& Hensler, G. 1996, in New light on galaxy evolution (Kluwer Academic Publishers), 23

Samland, M., Hensler, G., \& Theis, Ch. 1997, ApJ, 476, 544

Scalo, J. M. 1986, FCPh, 11, 1

Sommer-Larsen, J., \& Yoshii, Y. 1989, MNRAS, 238, 133

Sommer-Larsen, J., \& Yoshii, Y. 1990, MNRAS, 243, 468

Sommer-Larsen, J., Gelato, S., \& Vedel, H. 1999, ApJ, 519, 501

Spergel, D. N., Bean, R., Doré, O., et al. 2007, ApJS, 170, 377

Spite, M., Cayrel, R., Plez, B., et al. 2005, A\&A, 430, 655

Springel, V. 2005, MNRAS, 364, 1105

Springel, V., White, S. D. M., Tormen, G., \& Kauffmann, G. 2001, MNRAS, 328,726

Stephens, A. 1999, AJ, 117, 1771

Talbot, R. J. Jr., \& Arnett, W. D. 1971, ApJ, 170, 409

Talbot, R. J. Jr., \& Arnett, W. D. 1973a, ApJ, 186, 51

Talbot, R. J. Jr., \& Arnett, W. D. 1973b, ApJ, 186, 69

Talbot, R. J. Jr., \& Arnett, W. D. 1975, ApJ, 197, 551

Theis, C., Burkert, A., \& Hensler, G. 1992, A\&A, 265, 465

Timmes, F. X., Woosley, S. E., \& Weaver, T. A. 1995, ApJS, 98, 617

Tomkin, J., Woolf, V. M., Lambert, D. L., \& Lemke, M. 1995, AJ, 109, 2204

Tosi, M. 1988, A\&A, 197, 47

Tsujimoto, T., Yoshii, Y., Nomoto, K., \& Shigeyama, T. 1995, A\&A, 302, 704

van den Hoek, L. B., \& Groenewegen, M. A. T. 1997, A\&AS, 123, 305

Wechsler, R. H., Bullock, J. S., Primack, J. R., Kravtsov, A. V., \& Dekel, A. 2002, ApJ, 568, 52

Whelan, J., \& Iben, I. Jr. 1973, ApJ, 186, 1007

Woosley, S. E., \& Weaver, T. A. 1995, AIPC, 327, 365

Wyse, R. F. G., \& Gilmore, G. 1995, AJ, 110, 2771 